\title{
Weighted norm inequalities for multilinear singular integral operators and applications
}

\author{
Guoen $\mathrm{Hu}$ \\ Department of Applied Mathematics, Zhengzhou Information Science and Technology Institute \\ P.O. Box 1001-747, Zhengzhou 450002, China \\ E-mail: guoenxx@yahoo.com.cn
}

Chin-Cheng Lin

Department of Mathematics, National Central University

Chung-Li 320, Taiwan

E-mail: clin@math.ncu.edu.tw

\begin{abstract}
In this paper, weighted norm inequalities with $A_{p}$ weights are established for the multilinear singular integral operators whose kernels satisfy $L^{r^{\prime}}$-Hörmander regularity condition. As applications, we recover a weighted estimate for the multilinear Fourier multiplier obtained by $\mathrm{Fu}-$ jita and Tomita, and obtain several new weighted estimates for the multilinear Fourier multiplier as well.
\end{abstract}

Keywords. Multilinear singular integral operator; multilinear Fourier multiplier.

MR(2000) Subject Classification. 42B20, 42B30.

\section{Introduction.}

The study of the multilinear singular integral operators was originated by Coifman and Meyer in their celebrated work [3, 4]. Let $m \in \mathbb{N}$ and $K\left(x ; y_{1}, \ldots, y_{m}\right)$ be a locally integrable function defined away from the diagonal $x=y_{1}=y_{2}=\cdots=y_{m}$ in $\left(\mathbb{R}^{n}\right)^{m+1}$. An operator $T$, defined on $m$-fold products of $\mathscr{S}\left(\mathbb{R}^{n}\right)$ (Schwartz space) and taking values in the space of tempered distributions, is said to be an $m$-linear singular integral operator with kernel $K$ if $T$ is $m$-linear and satisfies that

$$
T\left(f_{1}, \ldots, f_{m}\right)(x)=\int_{\left(\mathbb{R}^{n}\right)^{m}} K\left(x ; y_{1}, \ldots, y_{m}\right) f_{1}\left(y_{1}\right) \ldots f_{m}\left(y_{m}\right) d \vec{y}
$$

Research of the first author was supported by National Natural Science Foundation of China under Grant \#10971228. Research of the second author was supported by National Science Council of Taiwan under Grant \#NSC 100-2115-M-008-002-MY3. 
for bounded functions $f_{1}, \ldots, f_{m}$ with compact supports, and $x \in \mathbb{R}^{n} \backslash \cup_{j=1}^{m} \operatorname{supp} f_{j}$, where $d \vec{y}=d y_{1} \ldots d y_{m}$. Operators of this type plays an important role in multilinear harmonic analysis. When $T$ is an $m$-linear Calderón-Zygmund operator; that is, $T$ is bounded from $L^{q_{1}}\left(\mathbb{R}^{n}\right) \times \cdots \times$ $L^{q_{m}}\left(\mathbb{R}^{n}\right)$ to $L^{q}\left(\mathbb{R}^{n}\right)$ for some $q_{1}, \ldots, q_{m} \in[1, \infty]$ and $q \in(0, \infty)$ with $1 / q=\sum_{1 \leq k \leq m} 1 / q_{k}$, and the associated kernel $K$ is an $m$-Calderón-Zygmund kernel, Grafakos and Torres [1] considered the endpoint estimate for $T$ on the space of type $L^{1}\left(\mathbb{R}^{n}\right) \times \cdots \times L^{1}\left(\mathbb{R}^{n}\right)$, and established a $T 1$ type theorem for the operator $T$. Grafakos and Kalton [8] proved that the multilinear CalderónZygmund operator is bounded from the products of Hardy spaces into Lebesgue spaces. See also [12, 13, 17] for more results on the multilinear Calderón-Zygmund operator.

Recently Anh and Duong [1] introduced a class of multilinear singular integral operators whose kernels satisfy that there exist two positive constant $r \in(1, \infty)$ and $\varrho \in(0,1]$ such that, for any ball $B$ and $x, x^{\prime} \in B$,

$$
\begin{aligned}
& \left(\int_{S_{j_{m}}(B)} \cdots \int_{S_{j_{1}}(B)}\left|K\left(x ; y_{1}, \ldots, y_{m}\right)-K\left(x^{\prime} ; y_{1}, \ldots, y_{m}\right)\right|^{r^{\prime}} d \vec{y}\right)^{1 / r^{\prime}} \\
& \quad \lesssim \frac{\left|x-x^{\prime}\right|^{\varrho}}{\left|2^{j^{*}} B\right|^{m / r+\varrho / n}}
\end{aligned}
$$

where $j_{1}, \ldots, j_{m}$ are integers with $\max _{1 \leq j \leq m} j_{k}>0$ and $j^{*}=\max _{1 \leq k \leq m} j_{k}$. Here and what follows, we denote by $r^{\prime}$ the index conjugate to $r$; that is, $r^{\prime}=r /(r-1)$ for $1 \leq r \leq \infty$. For a ball $B$, we denote $S_{N}(B):=2^{N} B \backslash 2^{N-1} B$ for $N \in \mathbb{N}$, and $S_{0}(B):=B$. Anh and Duong considered the weighted estimates with multiple weights for the multilinear singular integral operators when the associated kernels satisfy (1.2), and obtained several new weighted estimates for multilinear Fourier multiplier operators.

The purpose of this paper is to establish weighted norm inequalities for multilinear singular integral operators whose kernels satisfy certain $L^{r^{\prime}}$-Hörmander condition. Before stating our results, we first recall some notations.

A function $w$ is said to be a weight if it is nonnegative and locally integrable. Let $M$ denote the Hardy-Littlewood maximal operator. For $r \in(0, \infty)$, define $M_{r}$ to be the operator given by

$$
M_{r} f(x)=\left\{M\left(|f|^{r}\right)(x)\right\}^{1 / r}
$$

For a weight $w$, the weighted weak $L^{p}\left(\mathbb{R}^{n}\right)$ with respect to $w$ is defined as

$$
L^{p, \infty}\left(\mathbb{R}^{n}, w\right)=\left\{f:\|f\|_{L^{p, \infty}\left(\mathbb{R}^{n}, w\right)}<\infty\right\},
$$

where $\|f\|_{L^{p, \infty\left(\mathbb{R}^{n}, w\right)}}^{p}:=\sup _{\lambda>0} \lambda^{p} w\left(\left\{x \in \mathbb{R}^{n}:|f(x)|>\lambda\right\}\right)$.

A weight $w$ is said to belong to the Muckenhoupt class $A_{p}\left(\mathbb{R}^{n}\right), 1<p<\infty$, if

$$
\sup _{B}\left(\frac{1}{|B|} w(x) d x\right)\left(\frac{1}{|B|} \int_{B} w(x)^{1-p^{\prime}} d x\right)^{p-1}<\infty
$$


where the supremum is taken over all balls $B \subset \mathbb{R}^{n}$. A weight $w$ is said to belong to the class $A_{1}\left(\mathbb{R}^{n}\right)$ if, for any ball $B$,

$$
\frac{1}{|B|} \int_{B} w(x) d x \lesssim \inf _{x \in B} w(x)
$$

For the properties of $A_{p}\left(\mathbb{R}^{n}\right)$, we refer readers to [7].

The main result of this article is the following

Theorem 1.1. Let $T$ be an m-linear singular integral operator with kernel $K$ in the sense of (1.1). For $x, x^{\prime}, y_{1}, \ldots, y_{k} \in \mathbb{R}^{n}$, set

$$
W_{0}\left(x, y_{1}, \ldots, y_{m} ; x^{\prime}\right)=\left|K\left(x ; y_{1}, \ldots, y_{k}\right)-K\left(x^{\prime} ; y_{1}, \ldots, y_{m}\right)\right|,
$$

and for $y_{k}^{\prime} \in \mathbb{R}^{n}, 1 \leq k \leq m$,

$$
W_{k}\left(x, y_{1}, \ldots, y_{m} ; y_{k}^{\prime}\right)=\left|K\left(x ; y_{1}, \ldots, y_{k}\right)-K\left(x ; y_{1}, \ldots, y_{k}^{\prime}, \ldots, y_{m}\right)\right| .
$$

Let $r_{1}, \ldots, r_{m} \in[1, \infty)$. Suppose that

(i) for any $x \in \mathbb{R}^{n}$,

$$
\left.\sup _{R>0} R^{\frac{n}{r_{1}}+\cdots+\frac{n}{r_{m}}}\left(\int_{A_{R}^{x}} \ldots\left(\int_{A_{R}^{x}}\left|K\left(x ; y_{1}, \ldots, y_{m}\right)\right|^{r_{m}^{\prime}} d y_{m}\right)^{\frac{r_{m-1}^{\prime}}{r_{m}^{\prime}}} \ldots\right)^{\frac{r_{1}^{\prime}}{r_{2}^{\prime}}} d y_{1}\right)^{\frac{1}{r_{1}^{\prime}}}<\infty,
$$

where $A_{R}^{x}=\{y: R / 2 \leq|y-x| \leq 2 R\}$

(ii) for any ball $B$ and $x, x^{\prime} \in B$, and any $f_{1}, \ldots, f_{m}$ such that $\operatorname{supp} f_{k} \subset \mathbb{R}^{n} \backslash 4 B$ for some $1 \leq k \leq m$,

$$
\int_{\left(\mathbb{R}^{n}\right)^{m}}\left|W_{0}\left(x, y_{1}, \ldots, y_{m}, x^{\prime}\right)\right|\left|f_{1}\left(y_{1}\right) \ldots f_{m}\left(y_{m}\right)\right| d \vec{y} \lesssim \prod_{k=1}^{m}\left(M_{r_{k}} f_{k}(x)+M_{r_{k}} f_{k}\left(x^{\prime}\right)\right)
$$

(iii) for each integer $k$ with $1 \leq k \leq m$, and each ball $B$ with radial $R$, there exists a function $\mathrm{H}_{k, B}$, such that for function $f_{k}$ with $\operatorname{supp} f_{k} \subset B$ and any $x \in \mathbb{R}^{n} \backslash 4 B, y_{k}, y_{k}^{\prime} \in B$,

$$
\begin{aligned}
\int_{\left(\mathbb{R}^{n}\right)^{m}}\left|W_{k}\left(x, y_{1}, \ldots, y_{m} ; y_{k}\right)\right| \prod_{l=1}^{m}\left|f_{l}\left(y_{l}\right)\right| d \vec{y} \\
\lesssim \int_{\mathbb{R}^{n}}\left|f_{k}\left(y_{k}\right)\right| \mathrm{H}_{k, B}\left(x, y_{k}, y_{k}^{\prime}\right) d y_{k} \prod_{1 \leq l \leq m, l \neq k} M_{r_{l}} f_{l}(x),
\end{aligned}
$$

and for any integer $j_{0} \geq 3$,

$$
\left(\int_{S_{j_{0}}(B)}\left|\mathrm{H}_{k}\left(x, y_{k}, y_{k}^{\prime}\right)\right|^{r_{k}^{\prime}} d x\right)^{1 / r_{k}^{\prime}} \lesssim \frac{R^{\varrho}}{\left|2^{j_{0}} B\right|^{\frac{1}{r_{k}}+\varrho / n}},
$$

with @ a positive constant; 
(iv) $T$ is bounded from $L^{q_{1}}\left(\mathbb{R}^{n}\right) \times \cdots \times L^{q_{m}}\left(\mathbb{R}^{n}\right)$ to $L^{q, \infty}\left(\mathbb{R}^{n}\right)$ for some $q_{1}, \ldots, q_{m} \in[1, \infty]$ and $q \in(0, \infty)$ with $1 / q=\sum_{1 \leq k \leq m} 1 / q_{k}$.

We have the following weighted estimates for $T$.

(a) If for $1 \leq k \leq m, p_{k} \in\left(r_{k}, \infty\right]$ and the weight $w_{k} \in A_{p_{k} / r_{k}}\left(\mathbb{R}^{n}\right), p \in(0, \infty)$ such that $1 / p=\sum_{1 \leq k \leq m} 1 / p_{k}$, then

$$
\left\|T\left(f_{1}, \ldots, f_{m}\right)\right\|_{L^{p}\left(\mathbb{R}^{n}, \nu_{\vec{w}}\right)} \lesssim \prod_{k=1}^{m}\left\|f_{k}\right\|_{L^{p_{k}\left(\mathbb{R}^{n}, w_{k}\right)}}
$$

where $\nu_{\vec{w}}=\prod_{l=1}^{m} w_{l}^{p / p_{l}}$ (in case $p_{k}=\infty$, the terms $\left\|f_{k}\right\|_{L^{p_{k}}\left(\mathbb{R}^{n}, w_{k}\right)}$ on the right hand side of the inequality above is understood to be replaced by $\left.\left\|f_{k}\right\|_{L^{\infty}\left(\mathbb{R}^{n}\right)}\right)$;

(b) if for some $k$ with $1 \leq k \leq m, p_{k} \in\left(1, r_{k}^{\prime}\right)$, and $p_{l} \in\left(r_{l}, \infty\right]$ for any $1 \leq l \leq m$ and $l \neq k$, $w \in \cap_{1 \leq l \leq m, l \neq k} A_{p_{l} / r_{l}}\left(\mathbb{R}^{n}\right)$ and $w^{1-p_{k}^{\prime}} \in A_{p_{k}^{\prime} / r_{k}}\left(\mathbb{R}^{n}\right)$, then

$$
\left\|T\left(f_{1}, \ldots, f_{m}\right)\right\|_{L^{p}\left(\mathbb{R}^{n}, w\right)} \lesssim \prod_{l=1}^{m}\left\|f_{l}\right\|_{L^{p_{l}\left(\mathbb{R}^{n}, w\right)}} ;
$$

(c) if $p_{l} \in\left(r_{l}, \infty\right]$ for any $1 \leq l \leq m$ and $l \neq k, w^{r_{k}} \in A_{1}\left(\mathbb{R}^{n}\right)$, then

$$
\left\|T\left(f_{1}, \ldots, f_{m}\right)\right\|_{L^{p, \infty}\left(\mathbb{R}^{n}, w\right)} \lesssim\left\|f_{k}\right\|_{L^{1}\left(\mathbb{R}^{n}, w\right)} \prod_{1 \leq l \leq m, l \neq k}\left\|f_{l}\right\|_{L^{p} l\left(\mathbb{R}^{n}, w\right)}
$$

with $1 / p=1+\sum_{1 \leq l \leq m, l \neq k} 1 / p_{l}$.

We now consider the multilinear Fourier multiplier operator. Let $\sigma \in L^{\infty}\left(\mathbb{R}^{n m}\right)$. Define the $m$-linear Fourier multiplier operator $T_{\sigma}$ by

$$
\begin{aligned}
& T_{\sigma}\left(f_{1}, \ldots, f_{m}\right)(x)=\int_{\left(\mathbb{R}^{n}\right)^{m}} \exp \left(2 \pi i x\left(\xi_{1}+\cdots+\xi_{m}\right)\right) \\
& \times \sigma\left(\xi_{1}, \ldots, \xi_{m}\right) \widehat{f_{1}}\left(\xi_{1}\right) \ldots \widehat{f_{m}}\left(\xi_{m}\right) d \vec{\xi}
\end{aligned}
$$

for $f_{1}, \ldots, f_{m} \in \mathscr{S}\left(\mathbb{R}^{n}\right)$, where "^" denotes the Fourier transform. Coifman and Meyer [4] proved that if $\sigma \in C^{s}\left(\mathbb{R}^{n m} \backslash\{0\}\right)$ satisfies

$$
\left|\partial_{\xi_{1}}^{\alpha_{1}} \ldots \partial_{\xi_{m}}^{\alpha_{m}} \sigma\left(\xi_{1}, \ldots, \xi_{m}\right)\right| \leq C_{\alpha_{1}, \ldots, \alpha_{m}}\left(\left|\xi_{1}\right|+\cdots+\left|\xi_{m}\right|\right)^{-\left(\left|\alpha_{1}\right|+\cdots+\left|\alpha_{m}\right|\right)}
$$

for all $\left|\alpha_{1}\right|+\cdots+\left|\alpha_{N}\right| \leq s$ with $s \geq 2 m n+1$, then $T_{m}$ is bounded from $L^{p_{1}}\left(\mathbb{R}^{n}\right) \times \cdots \times L^{p_{m}}\left(\mathbb{R}^{n}\right)$ to $L^{p}\left(\mathbb{R}^{n}\right)$ for all $1<p_{1}, \ldots, p_{m}, p<\infty$ with $1 / p=\sum_{1 \leq k \leq m} 1 / p_{k}$. For the case of $s \geq n m+1$, Grafakos-Torres [11] and Kenig-Stein [15] (for $m=2$ ) improved Coifman and Meyer's multiplier 
theorem to the indices $1 / m \leq p \leq 1$ by using the multilinear Calderón-Zygmund operator theory. An important progress in this area was given by Tomita. Let $\Phi \in \mathscr{S}\left(\mathbb{R}^{n m}\right)$ satisfy

$$
\left\{\begin{array}{l}
\operatorname{supp} \Phi \subset\left\{\left(\xi_{1}, \ldots, \xi_{m}\right): 1 / 2 \leq \sum_{k=1}^{m}\left|\xi_{k}\right| \leq 2\right\} \\
\sum_{\kappa \in \mathbb{Z}} \Phi\left(2^{-\kappa} \xi_{1}, \ldots, 2^{-\kappa} \xi_{m}\right)=1 \quad \text { for all }\left(\xi_{1}, \ldots, \xi_{m}\right) \in \mathbb{R}^{n m} \backslash\{0\} .
\end{array}\right.
$$

Set

$$
\sigma_{\kappa}\left(\xi_{1}, \ldots, \xi_{m}\right)=\Phi\left(\xi_{1}, \ldots, \xi_{m}\right) \sigma\left(2^{\kappa} \xi_{1}, \ldots, 2^{\kappa} \xi_{m}\right) .
$$

Tomita 18 , proved that if

$$
\sup _{\kappa \in \mathbb{Z}} \int_{\left(\mathbb{R}^{n}\right)^{m}}\left(1+\left|\xi_{1}\right|^{2}+\cdots+\left|\xi_{m}\right|^{2}\right)^{s}\left|\widehat{\sigma}_{\kappa}\left(\xi_{1}, \ldots, \xi_{m}\right)\right|^{2} d \vec{\xi}<\infty
$$

for some $s>m n / 2$, then $T_{\sigma}$ is bounded from $L^{p_{1}}\left(\mathbb{R}^{n}\right) \times \cdots \times L^{p_{m}}\left(\mathbb{R}^{n}\right)$ to $L^{p}\left(\mathbb{R}^{n}\right)$ provided $p_{1}, \ldots, p_{m}, p \in(1, \infty)$ and $1 / p=\sum_{1 \leq k \leq m} 1 / p_{k}$. Grafakos and Si [10] considered the mapping properties from $L^{p_{1}}\left(\mathbb{R}^{n}\right) \times \cdots \times L^{p_{m}}\left(\mathbb{R}^{n}\right)$ to $L^{p}\left(\mathbb{R}^{n}\right)$ for $T_{\sigma}$ when $\sigma$ satisfies (1.6) and $p \leq 1$. Miyachi and Tomita 14] considered the problem to find minimal smoothness condition for bilinear Fourier multiplier. Let

$$
\left\|\sigma_{\kappa}\right\|_{W^{s_{1}}, \ldots, s_{m}\left(\mathbb{R}^{n m}\right)}=\left(\int_{\mathbb{R}^{n m}}\left\langle\xi_{1}\right\rangle^{2 s_{1}} \ldots\left\langle\xi_{m}\right\rangle^{2 s_{m}}\left|\widehat{\sigma}_{\kappa}\left(\xi_{1}, \ldots, \xi_{m}\right)\right|^{2} d \vec{\xi}\right)^{1 / 2}
$$

where $\left\langle\xi_{k}\right\rangle:=\left(1+\left|\xi_{k}\right|^{2}\right)^{1 / 2}$. Miyachi and Tomita [14 proved that if

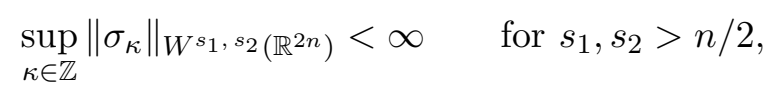

then $T_{\sigma}$ is is bounded from $L^{p_{1}}\left(\mathbb{R}^{n}\right) \times L^{p_{2}}\left(\mathbb{R}^{n}\right)$ to $L^{p}\left(\mathbb{R}^{n}\right)$ for any $p_{1}, p_{1} \in(1, \infty)$ and $p \geq 2 / 3$ with $1 / p=1 / p_{1}+1 / p_{2}$. Moreover, they also gives minimal smoothness condition for which $T_{\sigma}$ is bounded from $H^{p_{1}}\left(\mathbb{R}^{n}\right) \times H^{p_{2}}\left(\mathbb{R}^{n}\right)$ to $L^{p}\left(\mathbb{R}^{n}\right)$. It should be pointed out that the argument used in [14] applies to the case $m>2$. As an application of Theorem 1.1, we have

Theorem 1.2. Let $\sigma$ be a multiplier satisfying

$$
\sup _{\kappa \in \mathbb{Z}}\left\|\sigma_{\kappa}\right\|_{W^{s_{1}, \ldots, s_{m}}\left(\mathbb{R}^{m n}\right)}<\infty
$$

for $s_{1}, \ldots, s_{m} \in(n / 2, n]$ and $T_{\sigma}$ be the operator defined by (1.3). Set $t_{k}=n / s_{k}$. We have the following weighted estimates for $T_{\sigma}$.

(a) If $p_{k} \in\left(t_{k}, \infty\right]$ and the weight $w_{k} \in A_{p_{k} / t_{k}}\left(\mathbb{R}^{n}\right)$ for $1 \leq k \leq m$, and $p \in(0, \infty)$ such that $1 / p=\sum_{1 \leq k \leq m} 1 / p_{k}$, then

$$
\left\|T_{\sigma}\left(f_{1}, \ldots, f_{m}\right)\right\|_{L^{p}\left(\mathbb{R}^{n}, \nu_{\vec{w}}\right)} \lesssim \prod_{k=1}^{m}\left\|f_{k}\right\|_{L^{p_{k}\left(\mathbb{R}^{n}, w_{k}\right)}} ;
$$


(b) if $p_{k} \in\left(1, t_{k}^{\prime}\right)$ for some $k$ with $1 \leq k \leq m, p_{l} \in\left(r_{l}, \infty\right]$ for any $1 \leq l \leq m$ and $l \neq k$, $w \in \cap_{1 \leq l \leq m, l \neq k} A_{p_{l} / t_{l}}\left(\mathbb{R}^{n}\right)$ and $w^{1-p_{k}^{\prime}} \in A_{p_{k}^{\prime} / t_{k}}\left(\mathbb{R}^{n}\right)$, then

$$
\left\|T_{\sigma}\left(f_{1}, \ldots, f_{m}\right)\right\|_{L^{p}\left(\mathbb{R}^{n}, w\right)} \lesssim \prod_{l=1}^{m}\left\|f_{l}\right\|_{L^{p_{l}\left(\mathbb{R}^{n}, w\right)}} ;
$$

(c) if $p_{l} \in\left(r_{l}, \infty\right]$ for any $1 \leq l \leq m$ with $l \neq k$ and $w^{t_{k}} \in A_{1}\left(\mathbb{R}^{n}\right)$, then for $1 / p=1+$ $\sum_{1 \leq l \leq m, l \neq k} 1 / p_{k}$

$$
\left\|T_{\sigma}\left(f_{1}, \ldots, f_{m}\right)\right\|_{L^{p, \infty}\left(\mathbb{R}^{n}, w\right)}\left\|f_{k}\right\|_{L^{1}\left(\mathbb{R}^{n}, w\right)} \prod_{1 \leq l \leq m, l \neq k}\left\|f_{l}\right\|_{L^{p}\left(\mathbb{R}^{n}, w\right)}
$$

Remark. The conclusion (a) in Theorem 1.2 was proved in [6]. Here we give another simpler approach, which is of independent interest.

Throughout the article, $C$ always denotes a positive constant that may vary from line to line but remains independent of the main variables. We use the symbol $A \lesssim B$ to denote that there exists a positive constant $C$ such that $A \leq C B$. For any set $E \subset \mathbb{R}^{n}, \chi_{E}$ denotes its characteristic function. We use $B(x, R)$ to denote a ball centered at $x$ with radius $R$, and denote by $B_{R}$ the ball $B(0, R)$ for simplicity. For a ball $B \subset \mathbb{R}^{n}$ and $\lambda>0$, we use $\lambda B$ to denote the ball concentric with $B$ whose radius is $\lambda$ times of $B$ 's.

\section{Proof of Theorem 1.1 .}

Let $M^{\sharp}$ be the sharp maximal operator of Fefferman and Stein; that is, for a locally integrable function $f$,

$$
M^{\sharp} f(x)=\sup _{B \ni x} \frac{1}{|B|} \int_{B}\left|f(y)-V_{B}(f)\right| d y,
$$

where the supremum is taken over all balls containing $x$ and $V_{B}(f)$ denotes the mean value of $f$ on ball $B$. For a fixed $\delta>0$, let $M_{\delta}^{\sharp}$ be the operator defined by

$$
M_{\delta}^{\sharp}(f)(x)=\sup _{B \ni x} \inf _{c \in \mathbb{C}}\left(\frac{1}{|B|} \int_{B}|f(y)-c|^{\delta} d y\right)^{1 / \delta} .
$$

Lemma 2.1. Let $m \geq 1$ be an integer and $T$ be an $m$-linear singular integral operator associated with kernel $K$ in the sense of (1.1). Suppose that $T$ satisfies the assumptions (ii) and (iv) in Theorem [1.1. Let $r \in(0, \infty)$ such that $1 / r=\sum_{1 \leq k \leq m} 1 / r_{k}$, where $r_{k}$ 's are stated as in the assumption (ii) of Theorem 1.1. Then for any $\delta \in\left(0, \min \left\{q, r / r_{1}, \ldots, r / r_{m}\right\}\right)$,

$$
M_{\delta}^{\sharp}\left(T\left(f_{1}, \ldots, f_{m}\right)\right)(x) \lesssim \prod_{k=1}^{m} M_{\max \left\{q_{k}, r_{k}\right\}} f_{k}(x) .
$$


Proof. Let $x \in \mathbb{R}^{n}$ and $B$ be a ball containing $x$. Decompose $f_{k}(1 \leq k \leq m)$ as

$$
f_{k}(y)=f_{k}(y) \chi_{4 B}(y)+f_{k}(y) \chi_{\mathbb{R}^{n} \backslash 4 B}(y)=f_{k}^{1}(y)+f_{k}^{2}(y) .
$$

The fact that $T$ is bounded from $L^{q_{1}}\left(\mathbb{R}^{n}\right) \times \cdots \times L^{q_{m}}\left(\mathbb{R}^{n}\right)$ to $L^{q, \infty}\left(\mathbb{R}^{n}\right)$, together with the argument used in the proof of the Kolmogorov inequality, yields

$$
\begin{aligned}
\left(\frac{1}{|B|} \int_{B}\left|T\left(f_{1}^{1}, \ldots, f_{m}^{1}\right)(y)\right|^{\delta} d y\right)^{1 / \delta} & \lesssim \prod_{k=1}^{m}\left(\frac{1}{|B|} \int_{4 B}\left|f_{k}\left(y_{k}\right)\right|^{q_{k}} d y_{k}\right)^{1 / q_{k}} \\
& \lesssim \prod_{k=1}^{m} M_{q_{k}} f_{k}(x) .
\end{aligned}
$$

On the other hand, if $i_{1}, \ldots, i_{m} \in\{1,2\}$ and $i_{k}=2$ for some $k$ with $1 \leq k \leq m$, we then by assumption (ii) that

$$
\left|T\left(f_{1}^{i_{1}}, \ldots, f_{m}^{i_{m}}\right)(y)-T\left(f_{1}^{i_{1}}, \ldots, f_{m}^{i_{m}}\right)\left(y^{\prime}\right)\right| \lesssim \prod_{k=1}^{m}\left(M_{r_{k}} f_{k}(y)+M_{r_{k}} f_{k}\left(y^{\prime}\right)\right),
$$

for any $y^{\prime} \in B$ such that $\mid T\left(f_{1}^{i_{1}}, \ldots, f_{m}^{i_{m}}\left(y^{\prime}\right) \mid<\infty\right.$. It follows from [2] that

$$
M\left(M_{r_{k}} f_{k}\right)(z) \lesssim M_{r_{k}} f_{k}(z) \quad \text { for } z \in \mathbb{R}^{n} .
$$

Both estimates (2.1) and (2.2) lead to

$$
\begin{aligned}
\inf _{c \in \mathbb{C}}( & \left.\frac{1}{|B|} \int_{B}\left|T\left(f_{1}, \ldots, f_{m}\right)(y)-c\right|^{\delta} d y\right)^{\delta} \\
& \lesssim\left(\frac{1}{|B|^{2}} \int_{B} \int_{B}\left|T\left(f_{1}, \ldots, f_{m}\right)(y)-T\left(f_{1}, \ldots, f_{m}\right)\left(y^{\prime}\right)\right|^{\delta} d y d y^{\prime}\right)^{\delta} \\
\lesssim & \left(\frac{1}{|B|} \int_{B}\left|T\left(f_{1}^{1}, \ldots, f_{m}^{1}\right)(y)\right|^{\delta} d y\right)^{\delta} \\
& \quad+\sum_{i_{1}, \ldots, i_{m}}^{*}\left(\frac{1}{|B|^{2}} \int_{B} \int_{B}\left|T\left(f_{1}^{i_{1}}, \ldots, f_{m}^{i_{m}}\right)(y)-T\left(f_{1}^{i_{1}}, \ldots, f_{m}^{i_{m}}\right)\left(y^{\prime}\right)\right|^{\delta} d y d y^{\prime}\right)^{\delta} \\
\lesssim & \prod_{k=1}^{m} M_{q_{k}} f_{k}(x)+\left(\frac{1}{|B|} \int_{B} \prod_{k=1}^{m}\left\{M_{r_{k}} f_{k}(y)\right\}^{\delta} d y\right)^{1 / \delta} \\
\lesssim & \prod_{k=1}^{m} M_{q_{k}} f_{k}(x)+\prod_{k=1}^{m} M_{r_{k} \delta / r}\left(M_{r_{k}} f_{k}\right)(x) \\
\lesssim & \prod_{k=1}^{m} M_{\max \left\{q_{k}, r_{k}\right\}} f_{k}(x)
\end{aligned}
$$

where, for each term in the summation $\sum_{i_{1}, \ldots, i_{m}}^{*}$, the set of indices $\left\{i_{1}, \ldots, i_{m}\right\} \subset\{1,2\}$ and at least one $i_{k}=2(1 \leq k \leq m)$. This finishes the proof. 
Lemma 2.2. Let $p \in(0, \infty)$. If there exists $p_{0} \in(0, p)$ such that $\left\|M_{\delta} f\right\|_{L^{p_{0}, \infty}\left(\mathbb{R}^{n}\right)}<\infty$, then

$$
\left\|M_{\delta} f\right\|_{L^{p}\left(\mathbb{R}^{n}\right)} \lesssim\left\|M_{\delta}^{\sharp} f\right\|_{L^{p}\left(\mathbb{R}^{n}\right)} .
$$

Proof. Note that, for $\delta \in(0,1)$,

$$
\left\{M^{\sharp}\left(|h|^{\delta}\right)(x)\right\}^{1 / \delta} \lesssim M_{\delta}^{\sharp} h(x) .
$$

If $\left\|M_{\delta} f\right\|_{L^{p}\left(\mathbb{R}^{n}\right)}<\infty$ holds, then (2.3) follows from [7, Theorem 7.4.5 and Corollary 7.4.6]. On the other hand, for any positive real number $N$,

$$
\int_{0}^{N} \lambda^{p-1}\left|\left\{x \in \mathbb{R}^{n}: M_{\delta} f(x)>\lambda\right\}\right| d \delta \lesssim N^{p-p_{0}} \sup _{\lambda>0} \lambda^{p_{0}}\left|\left\{x \in \mathbb{R}^{n}: M_{\delta} f(x)>\lambda\right\}\right| .
$$

Thus, by the same argument in the proof of [7, Theorem 7.4.5], the lemma follows.

Lemma 2.3. Let $m \in \mathbb{N}$ and $T$ be an $m$-linear singular integral operator associated with kernel $K$ in the sense of (1.1). Suppose that

(1) T satisfies assumption (iii) in Theorem 1.1;

(2) $T$ is bounded from $L^{u_{1}}\left(\mathbb{R}^{n}\right) \times \cdots \times L^{u_{m}}\left(\mathbb{R}^{n}\right)$ to $L^{u, \infty}\left(\mathbb{R}^{n}\right)$ for some $u_{1}, \ldots, u_{m}$ with $u_{k} \in$ $\left[r_{k}, \infty\right](1 \leq k \leq m)$, and $u \in(0, \infty)$ with $1 / u=\sum_{1 \leq k \leq m} 1 / u_{k}$.

Then, for $p_{1}, \ldots, p_{m}$ such that $p_{k} \in\left[r_{k}, u_{k}\right], T$ is bounded from $L^{p_{1}}\left(\mathbb{R}^{n}\right) \times \cdots \times L^{p_{m}}\left(\mathbb{R}^{n}\right)$ to $L^{p, \infty}\left(\mathbb{R}^{n}\right)$ with $1 / p=\sum_{1 \leq k \leq m} 1 / p_{k}$.

Proof. Let $p_{k} \in\left[r_{k}, u_{k}\right](1 \leq k \leq m)$ and

$$
\left\|f_{1}\right\|_{L^{p_{1}\left(\mathbb{R}^{n}\right)}}=\cdots=\left\|f_{m}\right\|_{L^{p_{m}\left(\mathbb{R}^{n}\right)}}=1 .
$$

Our goal is to prove that there exists a constant depending only on $n, m$ and $p$ such that

$$
\left|\left\{x \in \mathbb{R}^{n}:\left|T\left(f_{1}, \ldots, f_{m}\right)(x)\right|>(C+1) \lambda\right\}\right| \lesssim \lambda^{-p} \quad \text { for all } \lambda>0 .
$$

To do this, we apply the Calderón-Zygmund decomposition. Given $\lambda>0$, applying the CalderónZygmund decomposition to $\left|f_{k}\right|^{p_{k}}$ at level $\lambda^{p}$, we obtain a sequence of cubes $\left\{Q_{k}^{j}\right\}_{j}$ satisfying

$$
\lambda^{p}<\frac{1}{\left|Q_{k}^{j}\right|} \int_{Q_{k}^{j}}\left|f_{k}(y)\right|^{p_{k}} d y \leq 2^{n} \lambda^{p}
$$

and

$$
\left|f_{k}(x)\right| \leq \lambda^{p / p_{k}} \quad \text { a.e. } x \in \mathbb{R}^{n} \backslash \cup_{j} Q_{k}^{j}
$$

Set

$$
\begin{aligned}
& f_{k}^{1}(y):=f_{k}(y) \chi_{\mathbb{R}^{n} \backslash \cup_{j} Q_{k}^{j}}(y)+\sum_{j} V_{Q_{k}^{j}}\left(f_{k}\right) \chi_{Q_{k}^{j}}(y), \\
& f_{k}^{2}(y):=f_{k}(y)-f_{k}^{1}(y)=\sum_{j} b_{k}^{j}(y)
\end{aligned}
$$


where $b_{k}^{j}(y)=\left(f_{k}(y)-V_{Q_{k}^{j}}\left(f_{k}\right)\right) \chi_{Q_{k}^{j}}(y)$. It is well known that $f_{k}^{1} \in L^{u_{k}}\left(\mathbb{R}^{n}\right)$ and

$$
\begin{aligned}
& \left\|f_{k}^{1}\right\|_{L^{u_{k}\left(\mathbb{R}^{n}\right)}} \lesssim \lambda^{p / p_{k}\left(1-p_{k} / u_{k}\right)}\left\|f_{k}\right\|_{L^{p_{k}\left(\mathbb{R}^{n}\right)}}^{p_{k} / u_{k}} \lesssim \lambda^{p / p_{k}-p / u_{k}}, \\
& \left\|f_{k}^{2}\right\|_{L^{p_{k}\left(\mathbb{R}^{n}\right)}} \lesssim\left\|f_{k}\right\|_{L^{p_{k}\left(\mathbb{R}^{n}\right)}} \lesssim 1
\end{aligned}
$$

Recall that $T$ is bounded from $L^{u_{1}}\left(\mathbb{R}^{n}\right) \times \ldots L^{u_{m}}\left(\mathbb{R}^{n}\right)$ to $L^{u, \infty}\left(\mathbb{R}^{n}\right)$. It follows that

$$
\left|\left\{x \in \mathbb{R}^{n}:\left|T\left(f_{1}^{1}, \ldots, f_{m}^{1}\right)(x)\right|>\lambda\right\}\right| \lesssim \lambda^{-u} \prod_{k=1}^{m}\left\|f_{k}^{1}\right\|_{L^{u_{k}\left(\mathbb{R}^{n}\right)}}^{u} \lesssim \lambda^{-p} .
$$

The proof of (2.4) is now reduced to proving

$$
\left|\left\{x \in \mathbb{R}^{n}: \sum_{i_{1}, \ldots, i_{m}}^{*}\left|T\left(f_{1}^{i_{1}}, \ldots, f_{m}^{i_{m}}\right)(x)\right|>C \lambda\right\}\right| \lesssim \lambda^{-p}
$$

where, for each term $T\left(f_{1}^{i_{1}}, \ldots, f_{m}^{i_{m}}\right)$ in the sum $\sum_{i_{1}, \ldots, i_{m}}^{*}$, each one of $i_{1}, \ldots, i_{m}$ is either 1 or 2 , and at least one $i_{k}=2$.

To prove (2.5), without loss of generality we may assume $i_{1}=2$. Let $\Omega=\cup_{k=1}^{m} \cup_{j} 8 B_{k}^{j}$, where $B_{k}^{j}$ is the smallest ball containing $Q_{k}^{j}$. For each $x \in \mathbb{R}^{n} \backslash \Omega$ and each fixed $j$, applying the vanishing moment of $b_{1}^{j}$ and the Hölder inequality, we write

$$
\begin{aligned}
\left|T\left(b_{1}^{j}, f_{2}^{i_{2}}, \ldots, f_{i_{m}}^{i_{m}}\right)(x)\right| & \\
\quad & \lesssim \int_{\left(\mathbb{R}^{n}\right)^{m}}\left|K\left(x ; y_{1}, \ldots, y_{m}\right)-K\left(x ; y_{1}^{j}, y_{2}, \ldots, y_{m}\right)\right|\left|b_{1}^{j}\left(y_{1}\right)\right| \prod_{k=2}^{m}\left|f_{k}^{i_{k}}\left(y_{k}\right)\right| d \vec{y} \\
\quad & \lesssim \int_{B_{1}^{j}}\left|b_{1}^{j}\left(y_{1}\right)\right| \mathrm{H}_{1, B_{1}^{j}}\left(x, y_{1}, y_{1}^{j}\right) d y_{1} \prod_{k=2}^{m} M_{r_{k}} f_{k}^{i_{k}}(x),
\end{aligned}
$$

where $y_{1}^{j}$ is the center of $B_{1}^{j}$. Let

$$
\mathrm{I}(x)=\sum_{j} \int_{B_{1}^{j}}\left|b_{1}^{j}\left(y_{1}\right)\right| \mathrm{H}_{1, B_{1}^{j}}\left(x, y_{1}, y_{1}^{j}\right) d y_{1} .
$$

We then have

$$
\mid T\left(f_{1}^{2}, f_{2}^{i_{2}}, \ldots, f_{m}^{i_{m}}(x) \mid \lesssim \mathrm{I}(x) \prod_{k=2}^{m} M_{r_{k}} f_{k}^{i_{k}}(x) .\right.
$$

Recall that, for any $k$ with $2 \leq k \leq m$,

$$
M_{r_{k}} f_{k}^{i_{k}}(x) \leq M_{r_{k}} f_{k}(x)+D \lambda^{p / p_{k}}
$$

for some constant $D$ depending only on $n$. Thus,

$$
\left|\left\{x \in \mathbb{R}^{n}: M f_{k}^{i_{k}}(x)>(D+1) \lambda^{p / p_{k}}\right\}\right| \lesssim \lambda^{-p} \int_{\mathbb{R}^{n}}\left|f_{k}(y)\right|^{p_{k}} d y \lesssim \lambda^{-p} .
$$


On the other hand, a trivial computation yields

$$
\begin{aligned}
\int_{\mathbb{R}^{n} \backslash \Omega} \mathrm{I}(x) d x & =\sum_{j} \sum_{l=3}^{\infty} \int_{S_{l}\left(B_{1}^{j}\right)} \int_{B_{1}^{j}} \int_{S_{l}\left(B_{1}^{j}\right)} \mathrm{H}_{1, B_{1}^{j}}\left(x, y_{1}, y_{1}^{\prime}\right) d x\left|b_{1}^{j}\left(y_{1}\right)\right| d y_{1} \\
& \lesssim \sum_{j} \sum_{l=3}^{\infty} \int_{B_{1}^{j}}\left(\int_{S_{l}\left(B_{1}^{j}\right)}\left|\mathrm{H}_{1, B_{1}^{j}}\left(x ; y_{1}, y_{1}^{j}\right)\right|^{r_{1}^{\prime}} d x\right)^{1 / r_{1}^{\prime}}\left|b_{1}^{j}\left(y_{1}\right)\right| d y_{1}\left|S_{l}\left(B_{1}^{j}\right)\right|^{1 / r_{1}} \\
& \lesssim \sum_{j} \int_{B_{1}^{j}}\left|b_{i}^{j}\left(y_{1}\right)\right| d y_{1}
\end{aligned}
$$

that implies

$$
\left|\left\{x \in \mathbb{R}^{n} \backslash \Omega: \mathrm{I}(x)>\lambda^{p / p_{1}}\right\}\right| \leq \lambda^{-p / p_{1}} \int_{\mathbb{R}^{n} \backslash \Omega} \mathrm{I}(x) d x \lesssim \lambda^{-p}
$$

Combining inequalities (2.7) $-(2.9)$, we obtain (2.5). This completes the proof of Lemma 2.3 .

We now are ready to show the main theorem.

Proof of Theorem 1.1. Since $T$ is bounded from $L^{q_{1}}\left(\mathbb{R}^{n}\right) \times \cdots \times L^{q_{m}}\left(\mathbb{R}^{n}\right)$ to $L^{q, \infty}\left(\mathbb{R}^{n}\right)$, it follows from Lemmas 2.1 and 2.2 that $T$ is bounded from $L^{p_{1}} \times \cdots \times L^{p_{m}}\left(\mathbb{R}^{n}\right)$ to $L^{p}\left(\mathbb{R}^{n}\right)$ with $1 / p=\sum_{1 \leq k \leq m} 1 / p_{k}$ provided $p_{k}>\max \left\{q_{k}, r_{k}\right\}$ for $k=1, \ldots, m$. Thus, by Lemma 2.3, $T$ is bounded from $L^{r_{1}}\left(\mathbb{R}^{n}\right) \times \cdots \times L^{r_{m}}\left(\mathbb{R}^{n}\right)$ to $L^{r, \infty}\left(\mathbb{R}^{n}\right)$ with $r \in(0, \infty)$ and $1 / r=\sum_{1 \leq k \leq m} 1 / r_{k}$. Hence, for $\delta \in\left(0, \min \left\{r / r_{1}, \ldots, r / r_{m}\right\}\right)$,

$$
M_{\delta}^{\sharp}\left(T\left(f_{1}, \ldots, f_{m}\right)\right)(x) \lesssim \prod_{k=1}^{m} M_{r_{k}} f_{k}(x) .
$$

Applying Lemma 2.2 again, we obtain the boundedness of $T$ from $L^{p_{1}}\left(\mathbb{R}^{n}\right) \times \cdots \times L^{p_{m}}\left(\mathbb{R}^{n}\right)$ to $L^{p}\left(\mathbb{R}^{n}\right)$ provided $p_{k} \in\left(r_{k}, \infty\right], 1 \leq k \leq m$, and $p \in(0, \infty)$ such that $1 / p=\sum_{1 \leq k \leq m} 1 / p_{k}$.

To prove conclusion (a), for $p_{k} \in\left(r_{k}, \infty\right]$ and $w_{k} \in A_{p_{k} / r_{k}}\left(\mathbb{R}^{n}\right), k=1, \ldots, m$, we claim that if $\delta$ is small enough, then for bounded functions $f_{1}, \ldots, f_{m}$ with compact supports,

$$
\left\|M_{\delta}\left(T\left(f_{1}, \ldots, f_{m}\right)\right)\right\|_{L^{p}\left(\mathbb{R}^{n}, \nu_{\vec{w}}\right)}<\infty .
$$

Once we prove the claim, conclusion (a) follows immediately from the inequalities (2.10) - (2.11) and the well known inequality of Córdoba and Fefferman [5].

The proof of (2.11) is fairly standard. We note that $\nu_{\vec{w}} \in A_{p / \delta}\left(\mathbb{R}^{n}\right)$ for $\delta$ small enough. If we take $R$ large enough such that $\cup_{k=1}^{m} \operatorname{supp} f_{k} \subset B_{R}$, then

$$
\begin{aligned}
\left\|M_{\delta}\left(T\left(f_{1}, \ldots, f_{m}\right)\right)\right\|_{L^{p}\left(\mathbb{R}^{n}, \nu_{\vec{w}}\right)}^{p} & \lesssim\left\|T\left(f_{1}, \ldots, f_{m}\right)\right\|_{L^{p}\left(\mathbb{R}^{n}, \nu_{\vec{w}}\right)}^{p} \\
& =\int_{B_{2 R}}\left|T\left(f_{1}, \ldots, f_{m}\right)(x)\right|^{p} \nu_{\vec{w}}(x) d x
\end{aligned}
$$




$$
+\int_{\mathbb{R}^{n} \backslash B_{2 R}}\left|T\left(f_{1}, \ldots, f_{m}\right)(x)\right|^{p} \nu_{\vec{w}}(x) d x
$$

It is obvious that $\int_{B_{2 R}}\left|T\left(f_{1}, \ldots, f_{m}\right)(x)\right|^{p} \nu_{\vec{w}}(x) d x<\infty$. On the other hand, the size condition (i) shows that, for $x \in \mathbb{R}^{n} \backslash B_{2 R}$,

$$
\begin{aligned}
\left|T\left(f_{1}, \ldots, f_{m}\right)(x)\right| & \lesssim \int_{\left\{\mathbb{R}^{m n}: \frac{|x|}{2} \leq\left|x-y_{k}\right| \leq 2|x|, k=1, \ldots, m\right\}}\left|K\left(x ; y_{1}, \ldots, y_{m}\right)\right|\left|f_{1}\left(y_{1}\right) \ldots f_{m}\left(y_{m}\right)\right| d \vec{y} \\
& \lesssim \prod_{k=1}^{m} M_{r_{k}} f_{k}(x) .
\end{aligned}
$$

Then, Hölder's inequality and the weighted boundedness of the Hardy-Littlewood maximal operator yield

$$
\int_{\mathbb{R}^{n} \backslash B_{2 R}}\left|T\left(f_{1}, \ldots, f_{m}\right)(x)\right|^{p} \nu_{\vec{w}}(x) d x \lesssim \prod_{k=1}^{m}\left\|f_{k}\right\|_{L^{p_{k}\left(\mathbb{R}^{n}, w_{k}\right)}}^{p} .
$$

This leads to our claim directly.

For conclusion (b), we consider the case $k=1$ only. Let $1<p_{1}<r_{1}^{\prime}, p_{l}>r_{l}$ for $2 \leq$ $l \leq m, w \in \cap_{l=2}^{m} A_{p_{l} / r_{l}}\left(\mathbb{R}^{n}\right)$ and $w^{1-p_{1}^{\prime}} \in A_{p_{1}^{\prime} / r_{1}}\left(\mathbb{R}^{n}\right)$. Choose points $\left(1 / p_{1}^{1}, \ldots, 1 / p_{m}^{1}, 1 / p^{1}\right), \ldots$, $\left(1 / p_{1}^{m+1}, \ldots, 1 / p_{m}^{m+1}, 1 / p^{m+1}\right)$, such that $1 / p^{j}=\sum_{1 \leq k \leq m} 1 / p_{k}^{j}$ for any $1 \leq j \leq m+1$, and

(1) $\left(1 / p_{1}, \ldots, 1 / p_{m}, 1 / p\right)$ is in the open convex hull of the points $\left(1 / p_{1}^{1}, \ldots, 1 / p_{m}^{1}, 1 / p^{1}\right), \ldots$, $\left(1 / p_{1}^{m+1}, \ldots, 1 / p_{m}^{m+1}, 1 / p^{m+1}\right)$;

(2) for each $i \in\{1, \ldots, m+1\}$, either $p_{l}^{i}>r_{l}$ for all $1 \leq l \leq m$ and $w \in \cap_{l=1}^{m} A_{p_{l}^{i} / r_{l}}\left(\mathbb{R}^{n}\right)$, or $1<p_{1}^{i}<r_{1}^{\prime}, p_{l}^{i}>r_{l}$ for $2 \leq l \leq m, w^{1-\left(p_{1}^{i}\right)^{\prime}} \in A_{\left(p_{1}^{i}\right)^{\prime} / r_{1}}\left(\mathbb{R}^{n}\right)$ and $w \in \cap_{l=2}^{m} A_{p_{l}^{i} / r_{l}}\left(\mathbb{R}^{n}\right)$.

Thus, by the multilinear Marcinkiewicz interpolation theorem (cf. [7]), it suffices to prove the boundedness of $T$ from $L^{p_{1}}\left(\mathbb{R}^{n}, w\right) \times \cdots \times L^{p_{m}}\left(\mathbb{R}^{n}, w\right)$ to $L^{p, \infty}\left(\mathbb{R}^{n}, w\right)$ whenever $p_{1} \in\left(1, r_{1}^{\prime}\right)$, $p_{l}>r_{l}$ for $2 \leq l \leq m, w^{1-p_{1}^{\prime}} \in A_{p_{1}^{\prime} / r_{1}}\left(\mathbb{R}^{n}\right)$ and $w \in A_{p_{k} / r_{k}}\left(\mathbb{R}^{n}\right)$ for $2 \leq k \leq m$.

For $f_{k} \in L^{p_{k}}\left(\mathbb{R}^{n}\right), 1 \leq k \leq m$, with

$$
\left\|f_{1}\right\|_{L^{p_{1}\left(\mathbb{R}^{n}, w\right)}}=\cdots=\left\|f_{m}\right\|_{L^{p_{m}\left(\mathbb{R}^{n}, w\right)}}=1,
$$

applying the weighted Calderón-Zygmund decomposition to $\left|f_{1}\right|^{p_{1}}$ at level $\lambda^{p}$, we obtain a sequence of cubes $\left\{Q_{1}^{j}\right\}_{j}$ such that

$$
\begin{gathered}
\lambda^{p} \leq \frac{1}{w\left(Q_{1}^{j}\right)} \int_{Q_{1}^{j}}\left|f_{1}(y)\right|^{p_{1}} w(y) d y \leq 2^{n} \lambda^{p}, \\
\left|f_{1}(x)\right| \leq \lambda^{p / p_{1}} \quad \text { a.e. } x \in \mathbb{R}^{n} \backslash \cup_{j} Q_{1}^{j} .
\end{gathered}
$$

Let $f_{1}^{1}, f_{1}^{2}$ be the functions given in the proof of Lemma 2.3 and $\Omega=\cup_{j} 8 B_{1}^{j}$, where $B_{1}^{j}$ is the ball circumscribed on $Q_{1}^{j}$. Then $w(\Omega) \lesssim \lambda^{-p}$. Since $w^{1-p_{1}^{\prime}} \in A_{p_{1}^{\prime} / r_{1}}\left(\mathbb{R}^{n}\right)$, we can choose $t_{1}$ large 
enough such that $w \in A_{t_{1} / r_{1}}\left(\mathbb{R}^{n}\right)$. Thus, by conclusion (a),

$$
\begin{aligned}
w\left(\left\{x \in \mathbb{R}^{n}:\left|T\left(f_{1}^{1}, f_{2}, \ldots, f_{m}\right)(x)\right|>\lambda\right\}\right) & \lesssim \lambda^{-t} \prod_{k=2}^{m}\left\|f_{k}^{1}\right\|_{L^{p_{k}\left(\mathbb{R}^{n}, w\right)}}^{t}\left\|f_{1}^{1}\right\|_{L^{t_{1}\left(\mathbb{R}^{n}, w\right)}}^{t} \\
& \lesssim \lambda^{-p}
\end{aligned}
$$

where $1 / t=1 / t_{1}+\sum_{2 \leq k \leq m} 1 / p_{k}$.

To estimate $T\left(f_{1}^{2}, f_{2}, \ldots, f_{m}\right)(x)$, we employ the idea used in [16]. Similar to the proof of Lemma 2.3, for $x \in \mathbb{R}^{n} \backslash \Omega$,

$$
\left|T\left(f_{1}^{2}, f_{2}, \ldots, f_{m}\right)(x)\right| \lesssim \sum_{j} \int_{B_{1}^{j}} \mathrm{H}_{1, B_{1}^{j}}\left(x ; y_{1}, y_{1}^{j}\right)\left|b_{1}^{j}\left(y_{1}\right)\right| d y_{1} \prod_{k=2}^{m} M_{r_{k}} f_{k}(x) .
$$

By the weighted boundedness of the Hardy-Littlewood maximal operator, if $w \in A_{p_{k} / r_{k}}\left(\mathbb{R}^{n}\right)$, then

$$
w\left(\left\{x \in \mathbb{R}^{n}: M_{r_{k}} f_{k}(x)>(D+1) \lambda^{p / p_{k}}\right\}\right) \lesssim \lambda^{-p} \int_{\mathbb{R}^{n}}\left|f_{k}(y)\right|^{p_{k}} w(y) d y
$$

Let $\mathrm{I}(x)$ be given in (2.6). A duality argument shows that if $w^{1-p_{1}^{\prime}} \in A_{p_{1}^{\prime} / r_{1}}\left(\mathbb{R}^{n}\right)$ and $F \in$ $L^{p_{1}^{\prime}}\left(\mathbb{R}^{n} \backslash \Omega, w\right)$ with $\|F\|_{L^{p_{1}^{\prime}}\left(\mathbb{R}^{n} \backslash \Omega, w^{\left.1-p_{1}^{\prime}\right)}\right.} \leq 1$, then

$$
\begin{aligned}
\left|\int_{\mathbb{R}^{n} \backslash \Omega} \mathrm{I}(x) F(x) d x\right| & \lesssim \sum_{j} \int_{\mathbb{R}^{n} \backslash \Omega} \int_{B_{1}^{j}} \mathrm{H}_{1, B_{1}^{j}}\left(x ; y_{1}, y_{1}^{j}\right)\left|b_{1}^{j}\left(y_{1}\right)\right| d y_{1} F(x) d x \\
& \lesssim \sum_{j} \sum_{l=3}^{\infty} \int_{B_{1}^{j}}\left(\int_{S_{l}\left(B_{1}^{j}\right)}\left|\mathrm{H}_{1, B_{1}^{j}}\left(x ; y_{1}, y_{1}^{j}\right)\right|^{r_{1}^{\prime}} d x\right)^{1 / r_{1}^{\prime}}\left|b_{1}^{j}\left(y_{1}\right)\right| d y_{1} \\
& \quad \times \inf _{y \in B_{1}^{j}} M_{r_{1}} F(y)\left|S_{l}\left(B_{1}^{j}\right)\right|^{1 / r_{1}} \\
& \lesssim \int_{\mathbb{R}^{n}}\left|f_{1}(y)\right| M_{r_{1}} F(y) d y \\
& \lesssim 1 .
\end{aligned}
$$

Therefore,

$$
w\left(\left\{x \in \mathbb{R}^{n} \backslash \Omega: \mathrm{I}(x)>\lambda^{p / p_{1}}\right) \lesssim \lambda^{-p} \int_{\mathbb{R}^{n} \backslash \Omega} \mathrm{I}(x)^{p_{1}} w(x) d x \lesssim \lambda^{-p} .\right.
$$

Estimates (2.12) - (2.14) give us

$$
w\left(\left\{x \in \mathbb{R}^{n}:\left|T\left(f_{1}, \ldots, f_{m}\right)\right|>C \lambda\right\}\right) \lesssim \lambda^{-p} .
$$

We turn the attention to conclusion (c), and consider the case $k=1$ only. Recall that $w^{r_{1}} \in A_{1}\left(\mathbb{R}^{n}\right)$ implies that $w \in A_{r}\left(\mathbb{R}^{n}\right)$ for any $r \geq 1$. A computation shows that

$$
\int_{\mathbb{R}^{n} \backslash \Omega} \mathrm{I}(x) w(x) d x \lesssim \sum_{j} \sum_{l=3}^{\infty} \int_{B_{1}^{j}}\left(\int_{S_{l}\left(B_{1}^{j}\right)}\left|\mathrm{H}_{1, B_{1}^{j}}\left(x ; y_{1}, y_{1}^{j}\right)\right|^{r_{1}^{\prime}} d x\right)^{1 / r_{1}^{\prime}}\left|b_{1}^{j}\left(y_{1}\right)\right| d y_{1}
$$




$$
\begin{gathered}
\times\left(\int_{S_{l}\left(B_{1}^{j}\right)} w(x)^{r_{1}} d x\right)^{1 / r_{1}} \\
\lesssim \sum_{j} \int_{B_{1}^{j}}\left|b_{i}^{j}\left(y_{1}\right)\right| w(y) d y_{1},
\end{gathered}
$$

which implies conclusion (c) by applying the Calderón-Zygmund decomposition and the estimates used in the proof of conclusion (b).

\section{Proof of Theorem 1.2 .}

We start with several preliminary lemmas.

Lemma 3.1. Let $\sigma_{\kappa}$ be defined in (1.5), $q_{1}, \ldots, q_{m} \in[2, \infty)$, and $s_{1}, \ldots, s_{m} \geq 0$. Then

$$
\begin{aligned}
& \left.\left(\int_{\mathbb{R}^{n}} \ldots\left(\int_{\mathbb{R}^{n}}\left|\widehat{\sigma}_{\kappa}\left(\xi_{1}, \ldots \xi_{m}\right)\right|^{q_{1}}\left\langle\xi_{1}\right\rangle^{s_{1}} d \xi_{1}\right)^{q_{2} / q_{1}}\left\langle\xi_{2}\right\rangle^{s_{2}} d \xi_{2}\right)^{q_{3} / q_{2}} \ldots\left\langle\xi_{m}\right\rangle^{s_{m}} d \xi_{m}\right)^{1 / q_{m}} \\
& \quad \lesssim\left\|\sigma_{\kappa}\right\|_{W^{s_{1} / q_{1}, \ldots, s_{m} / q_{m}}\left(\mathbb{R}^{m n}\right)} .
\end{aligned}
$$

For the proof of Lemma 3.1, see Appendix A in [6].

Lemma 3.2. Let $s_{1}, \ldots, s_{m} \in \mathbb{R}$, and $\alpha_{1} \ldots, \alpha_{m} \in \mathbb{Z}_{+}^{n}$ be multi-indices. Set

$$
\zeta_{\kappa}^{\alpha_{1}, \ldots, \alpha_{m}}\left(\xi_{1}, \ldots, \xi_{m}\right):=\xi_{1}^{\alpha_{1}} \ldots \xi_{m}^{\alpha_{m}} \sigma_{\kappa}\left(\xi_{1}, \ldots, \xi_{m}\right)
$$

Then

$$
\left\|\zeta_{\kappa}^{\alpha_{1}, \ldots, \alpha_{m}}\right\|_{W^{s_{1}, \ldots, s_{m}}\left(\mathbb{R}^{m n}\right)} \lesssim \sup _{l \in \mathbb{Z}}\left\|\sigma_{l}\right\|_{W^{s_{1}, \ldots, s_{m}}\left(\mathbb{R}^{m n}\right)}
$$

This lemma was given in [14, Remark 2.5].

Let $\sigma \in L^{\infty}\left(\mathbb{R}^{m n}\right)$ and $\Phi \in \mathscr{S}\left(\mathbb{R}^{m n}\right)$ satisfy (1.4). Define

$$
\widetilde{\sigma}_{\kappa}\left(\xi_{1}, \ldots, \xi_{m}\right)=\Phi\left(2^{-\kappa} \xi_{1}, \ldots, 2^{-\kappa} \xi_{m}\right) \sigma\left(\xi_{1}, \ldots, \xi_{m}\right) .
$$

Then

$$
\widetilde{\sigma}_{\kappa}\left(\xi_{1}, \ldots, \xi_{m}\right)=\sigma_{\kappa}\left(2^{-\kappa} \xi_{1}, \ldots, 2^{-\kappa} \xi_{m}\right)
$$

and

$$
\mathcal{F}^{-1} \widetilde{\sigma}_{\kappa}\left(\xi_{1}, \ldots, \xi_{m}\right)=2^{\kappa n m} \mathcal{F}^{-1} \sigma_{\kappa}\left(2^{\kappa} \xi_{1}, \ldots, 2^{\kappa} \xi_{m}\right),
$$

where $\mathcal{F}^{-1}$ denotes the inverse Fourier transform. For an integer $k$ with $1 \leq k \leq m$ and $x, y_{1}, \ldots, y_{m}, y_{k}^{\prime}, x^{\prime} \in \mathbb{R}^{n}$, let

$$
\begin{gathered}
W_{0, \kappa}\left(x, y_{1}, \ldots, y_{m} ; x^{\prime}\right)=\mathcal{F}^{-1} \widetilde{\sigma}_{\kappa}\left(x-y_{1}, \ldots, x-y_{m}\right)-\mathcal{F}^{-1} \widetilde{\sigma}_{\kappa}\left(x^{\prime}-y_{1}, \ldots, x^{\prime}-y_{m}\right), \\
W_{k, \kappa}\left(x, y_{1}, \ldots, y_{m} ; y_{k}^{\prime}\right)=\mathcal{F}^{-1} \widetilde{\sigma}_{\kappa}\left(x-y_{1}, \ldots, x-y_{m}\right)-\mathcal{F}^{-1} \widetilde{\sigma}_{\kappa}\left(x-y_{1}, \ldots, x-y_{k}^{\prime}, \ldots, x-y_{m}\right) .
\end{gathered}
$$


Also, for $r_{1}, \ldots, r_{m} \in(1, \infty)$, write

$$
\begin{aligned}
A_{k, \kappa}=( & \int_{S_{j_{0}}(B)}\left(\int _ { E _ { j _ { 1 } } ^ { R } ( x ) } \ldots \left(\int _ { E _ { j _ { k - 1 } } ^ { R } ( x ) } \left(\int _ { E _ { j _ { k + 1 } } ^ { R } ( x ) } \ldots \left(\int_{E_{j_{m}}^{R}(x)}\right.\right.\right.\right. \\
& \left.\left.\left.\left.\left.\left|W_{k, \kappa}\left(x, y_{1}, \ldots, y_{m} ; y_{k}^{\prime}\right)\right|^{r_{m}^{\prime}} d y_{m}\right)^{\frac{r_{m-1}^{\prime}}{r_{m}^{\prime}}} \ldots d y_{k+1}\right)^{\frac{r_{k}^{\prime}}{r_{k+1}^{\prime}}} d y_{k-1}\right)^{\frac{r_{k-2}^{\prime}}{r_{k-1}^{\prime}}} \ldots d y_{1}\right)^{\frac{r_{k}^{\prime}}{r_{1}^{\prime}}} d x\right)^{\frac{1}{r_{k}^{\prime}}},
\end{aligned}
$$

where $E_{0}^{R}(x)=B(x, R)$ and $E_{j}^{R}(x)=2^{j} B(x, R) \backslash 2^{j-1} B(x, R)$ for $j \in \mathbb{N}$.

Lemma 3.3. Let $m$ and $k$ be positive integers with $k \leq m, \sigma$ be a multiplier satisfying (1.7) for some $s_{1}, \ldots, s_{m} \in(n / 2, n]$, and $r_{1}, \ldots, r_{m} \in(1,2]$. Then, for any ball $B$ with radial $R$, $y_{k}, y_{k}^{\prime} \in \frac{1}{4} B, j_{0} \in \mathbb{N}$ and nonnegative integers $j_{1}, \ldots, j_{k-1}, j_{k+1}, \ldots, j_{m}$,

$$
A_{k, \kappa} \lesssim \frac{R 2^{-\kappa\left(s_{1}+\cdots+s_{m}-n / r_{1}-\cdots-n / r_{m}-1\right)}}{\left|2^{j_{0}} B\right|^{s_{k} / n} \prod_{1 \leq i \leq m, i \neq k}\left(2^{j_{i}} R\right)^{s_{i}}} \quad \text { if } \quad 2^{\kappa} R<1 .
$$

Proof. We prove (3.1) for $k=1$ only. Let $B_{R}=B(0, R)$. Step 1: we first consider the case that all of $j_{0}, j_{2}, \ldots, j_{m}$ are positive. Write

$$
\begin{aligned}
& \left|\mathcal{F}^{-1} \widetilde{\sigma}_{\kappa}\left(z_{1}, z_{2}, \ldots, z_{m}\right)-\mathcal{F}^{-1} \widetilde{\sigma}_{\kappa}\left(z_{1}+y_{1}-y_{1}^{\prime}, z_{2}, \ldots, z_{m}\right)\right| \\
& \quad=2^{\kappa n m}\left|\mathcal{F}^{-1} \sigma_{\kappa}\left(2^{\kappa} z_{1}, \ldots, 2^{\kappa} z_{m}\right)-\mathcal{F}^{-1} \sigma_{\kappa}\left(2^{\kappa} z_{1}+2^{\kappa}\left(y_{1}-y_{1}^{\prime}\right), 2^{\kappa} z_{2}, \ldots, 2^{\kappa} z_{m}\right)\right| \\
& \quad \leq 2^{\kappa n m} \sum_{|\alpha|=1}\left|2^{\kappa}\left(y_{1}-y_{1}^{\prime}\right)\right|^{\alpha} \int_{0}^{1}\left|\partial^{\alpha, 0, \ldots, 0} \mathcal{F}^{-1} \sigma_{\kappa}\left(2^{\kappa}\left(z_{1}+\theta\left(y_{1}-y_{1}^{\prime}\right)\right), 2^{\kappa} z_{2} \ldots, 2^{\kappa} z_{m}\right)\right| d \theta .
\end{aligned}
$$

Let

$$
\phi_{\kappa, y_{1}-y_{1}^{\prime}}\left(\theta ; z_{1}, \ldots, z_{m}\right)=\partial^{\alpha, 0, \ldots, 0} \mathcal{F}^{-1} \sigma_{\kappa}\left(2^{\kappa}\left(z_{1}+\theta\left(y_{1}-y_{1}^{\prime}\right)\right), 2^{\kappa} z_{2} \ldots, 2^{\kappa} z_{m}\right) \mid .
$$

By the Minkowsky inequality, Lemmas 3.1 and 3.2 .

$$
\begin{aligned}
A_{1, \kappa} \lesssim \sum_{|\alpha|=1} & \left(\int _ { C _ { j _ { 0 } } } \left(\int_{S_{j_{2}}\left(B_{R}\right)} \ldots\left(\int_{S_{j_{m}}\left(B_{R}\right)}\left(\int_{0}^{1}\left|\phi_{\kappa, y_{1}-y_{1}^{\prime}}\left(\theta ; z_{1}, \ldots, z_{m}\right)\right| d \theta\right)^{r_{m}^{\prime}} d z_{m}\right)^{\frac{r_{m-1}^{\prime}}{r_{m}^{\prime}}}\right.\right. \\
& \left.\ldots)^{\frac{r_{1}^{\prime}}{r_{2}^{\prime}}} d z_{1}\right)^{\frac{1}{r_{1}^{\prime}}} 2^{\kappa n m} 2^{\kappa} R \\
& \lesssim \sum_{|\alpha|=1} \int_{0}^{1}\left(\int _ { C _ { j _ { 0 } } } \left(\int_{S_{j_{2}}\left(B_{R}\right)} \ldots\left(\int_{S_{j_{m}}\left(B_{R}\right)}\left|\phi_{\kappa, y_{1}-y_{1}^{\prime}}\left(\theta ; z_{1}, \ldots, z_{m}\right)\right|^{r_{m}^{\prime}} d z_{m}\right)^{\frac{r_{m-1}^{\prime}}{r_{m}^{\prime}}}\right.\right. \\
& \left.\ldots)^{\frac{r_{1}^{\prime}}{r_{2}^{\prime}}} d z_{1}\right)^{\frac{1}{r_{1}^{\prime}}} d \theta 2^{\kappa n m} 2^{\kappa} R \\
\lesssim & \sum_{|\alpha|=1}\left(\int _ { C _ { j _ { 0 } } } \left(\int_{S_{j_{2}}\left(B_{R}\right)} \ldots\left(\int_{S_{j_{m}}\left(B_{R}\right)}\left|\partial^{\alpha, 0, \ldots, 0} \mathcal{F}^{-1} \sigma_{\kappa}\left(2^{\kappa} z_{1}, \ldots, 2^{\kappa} z_{m}\right)\right|^{r_{m}^{\prime}} d z_{m}\right)^{\frac{r_{m-1}^{\prime}}{r_{m}^{\prime}}}\right.\right.
\end{aligned}
$$




$$
\begin{aligned}
& \left.\ldots)^{\frac{r_{1}^{\prime}}{r_{2}^{\prime}}} d z_{1}\right)^{\frac{1}{r_{1}^{\prime}}} 2^{\kappa n m} 2^{\kappa} R \\
& \lesssim \sum_{|\alpha|=1}\left(\int _ { C _ { j _ { 0 } } } \left(\int_{S_{j_{2}}\left(B_{R}\right)} \ldots\left(\int_{S_{j_{m}}\left(B_{R}\right)}\left|\partial^{\alpha, 0, \ldots, 0} \mathcal{F}^{-1} \sigma_{\kappa}\left(2^{\kappa} z_{1}, \ldots, 2^{\kappa} z_{m}\right)\right|^{r_{m}^{\prime}}\left|z_{m}\right|_{m}^{r_{m}^{\prime} s_{m}} d z_{m}\right)^{\frac{r_{m-1}^{\prime}}{r_{m}^{\prime}}}\right.\right. \\
& \left.\left.\ldots\left|z_{2}\right|^{r_{2}^{\prime} s_{2}} d z_{2}\right)^{\frac{r_{1}^{\prime}}{r_{2}^{\prime}}}\left|z_{1}\right|^{r_{1}^{\prime} s_{1}} d z_{1}\right)^{\frac{1}{r_{1}^{\prime}}} 2^{\kappa} R\left(2^{j_{0}} R\right)^{-s_{1}} \prod_{i=2}^{m}\left(2^{j_{i}} R\right)^{-s_{i}} 2^{\kappa m n} \\
& \lesssim \sum_{|\alpha|=1}\left(\int _ { C _ { j _ { 0 } } } \left(\int_{S_{j_{2}}\left(B_{R}\right)} \ldots\left(\int_{S_{j_{m}}\left(B_{R}\right)}\left|\mathcal{F}^{-1}\left(\xi_{1}^{\alpha} \sigma_{\kappa}\right)\left(2^{\kappa} z_{1}, \ldots, 2^{\kappa} z_{m}\right)\right|^{r_{m}^{\prime}}\left|z_{m}\right|^{r_{m}^{\prime} s_{m}} d z_{m}\right)^{\frac{r_{m-1}^{\prime}}{r_{m}^{\prime}}}\right.\right. \\
& \left.\left.\quad \ldots\left|z_{2}\right|^{r_{2}^{\prime} s_{2}} d z_{2}\right)^{\frac{r_{1}^{\prime}}{r_{2}^{\prime}}}\left|z_{1}\right|^{r_{1}^{\prime} s_{1}} d z_{1}\right)^{\frac{1}{r_{1}^{\prime}}} 2^{\kappa} R\left(2^{j_{0}} R\right)^{-s_{1}} \prod_{i=2}^{m}\left(2^{j_{i}} R\right)^{-s_{i}} 2^{\kappa m n} \\
& \lesssim \sum_{|\alpha|=1}\left\|\xi_{1}^{\alpha} \sigma_{\kappa}\right\|_{W^{s_{1}, \ldots, s_{m}}\left(\mathbb{R}^{m n}\right)^{\kappa}} 2^{\kappa}\left(2^{j_{0}} R\right)^{-s_{1}} \prod_{i=2}^{m}\left(2^{j_{i}} R\right)^{-s_{i}} 2^{-\kappa\left(s_{1}+\cdots+s_{m}-n / r_{1}-\cdots-n / r_{m}\right)} \\
& \lesssim 2^{\kappa} R\left(2^{j_{0}} R\right)^{-s_{1}} \prod_{i=2}^{m}\left(2^{j_{i}} R\right)^{-s_{i}} 2^{-\kappa\left(s_{1}+\cdots+s_{m}-n / r_{1}-\cdots-n / r_{m}\right)}
\end{aligned}
$$

where $C_{j_{0}}=\left\{z: 2^{j_{0}-2} R \leq|z| \leq 2^{j_{0}+1} R\right\}$.

Step 2: if $\min \left\{j_{0}, j_{2}, \ldots, j_{m}\right\}=0$, for example, $j_{l+1}=\cdots=j_{m}=0$ and $j_{k} \geq 1$ for $2 \leq k \leq l$, then, for index $\alpha \in \mathbb{Z}_{+}^{n}$ and $2^{\kappa} R<1$,

$$
\begin{aligned}
& \left(\int _ { C _ { j _ { 0 } } } \left(\int_{S_{j_{2}}\left(B_{R}\right)} \cdots\left(\int_{S_{j_{m}}\left(B_{R}\right)}\left|\partial^{\alpha, 0, \ldots, 0} \mathcal{F}^{-1} \sigma_{\kappa}\left(2^{\kappa} z_{1}, \ldots, 2^{\kappa} z_{m}\right)\right|^{r_{m}^{\prime}} d z_{m}\right)^{\frac{r_{m-1}^{\prime}}{r_{m}^{\prime}}} d z_{m-1}\right.\right. \\
& \left.\ldots)^{\frac{r_{1}^{\prime}}{r_{2}^{\prime}}} d z_{1}\right)^{\frac{1}{r_{1}^{\prime}}} 2^{\kappa n m} 2^{\kappa} R \\
& \lesssim\left(\int _ { C _ { j _ { 0 } } } \left(\int _ { S _ { j _ { 2 } } ( B _ { R } ) } \ldots \left(\int _ { S _ { j _ { l } } ( B _ { R } ) } \left(\int_{B_{R}} \ldots\left(\int_{B_{R}}\left|\mathcal{F}^{-1}\left(\xi_{1}^{\alpha} \sigma_{\kappa}\right)\left(2^{\kappa} z_{1}, \ldots, 2^{\kappa} z_{m}\right)\right|^{r_{m}^{\prime}} d z_{m}\right)^{\frac{r_{m-1}^{\prime}}{r_{m^{\prime}}}}\right.\right.\right.\right. \\
& \left.\left.\left.\ldots)^{\frac{r_{l}^{\prime}}{r_{l+1}^{\prime}}}\left|z_{l}\right|^{r_{l}^{\prime} s_{l}} d z_{l}\right) \ldots\right)^{\frac{r_{1}^{\prime}}{r_{2}}}\left|z_{1}\right|^{r_{1}^{\prime} s_{1}} d z_{1}\right)^{\frac{1}{r_{1}^{\prime}}} 2^{\kappa} R\left(2^{j_{0}} R\right)^{-s_{1}} \prod_{i=2}^{l}\left(2^{j_{i}} R\right)^{-s_{i}} 2^{\kappa m n} \\
& \lesssim 2^{\kappa} R\left(2^{j_{0}} R\right)^{-s_{1}} \prod_{i=2}^{l}\left(2^{j_{i}} R\right)^{-s_{i}} 2^{-\kappa\left(s_{1}+\cdots+s_{l}-n / r_{1}-\cdots-n / r_{m}\right)} \\
& \lesssim 2^{\kappa} R\left(2^{j_{0}} R\right)^{-s_{1}} \prod_{i=2}^{l}\left(2^{j_{i}} R\right)^{-s_{i}} R^{-s_{l+1}-\cdots-s_{m}} 2^{-\kappa\left(s_{1}+\cdots+s_{m}-n / r_{1}-\cdots-n / r_{m}\right)} .
\end{aligned}
$$

This together with the argument in step 1 shows that, for $j_{2}, \ldots, j_{l}$ positive and $j_{l+1}=\cdots=$ $j_{m}=0$, inequality (3.1) holds $k=1$. 
Lemma 3.4. Let $\sigma$ be a multiplier satisfying (1.7) for $s_{1}, \ldots, s_{m} \in(n / 2, n]$, and $r_{1}, \ldots, r_{m} \in$ $(1,2]$ with $r_{l}>n / s_{l}$ for $1 \leq l \leq m$. Then, for each $\kappa \in \mathbb{Z}$ and integer $k$ with $1 \leq k \leq m$, there exists a function $\mathrm{H}_{k}^{\kappa}$ such that, for any ball $B$ with radial $R$, any function $f_{k}$ with $\operatorname{supp} f_{k} \subset B$, and any $x \in \mathbb{R}^{n} \backslash 4 B, y_{k}, y_{k}^{\prime} \in B$,

$$
\begin{aligned}
\int_{\left(\mathbb{R}^{n}\right)^{m}}\left|W_{k, \kappa}\left(x, y_{1}, \ldots, y_{m} ; y_{k}\right)\right| \prod_{l=1}^{m}\left|f_{l}\left(y_{l}\right)\right| d \vec{y} \\
\quad \lesssim \int_{\mathbb{R}^{n}}\left|f_{k}\left(y_{k}\right)\right| \mathrm{H}_{k}^{\kappa}\left(x, y_{k}, y_{k}^{\prime}\right) d y_{k} \prod_{1 \leq l \leq m, l \neq k} M_{r_{l}} f_{l}(x)
\end{aligned}
$$

and

$$
\left(\int_{S_{j_{0}}(B)}\left|\mathrm{H}_{k}^{\kappa}\left(x, y_{k}, y_{k}^{\prime}\right)\right|^{r_{k}^{\prime}} d x\right)^{1 / r_{k}^{\prime}} \lesssim \frac{2^{-\kappa\left(s_{k}-n / r_{k}\right)}}{\left(2^{j_{0}} R\right)^{s_{k}}} \quad \text { for integer } j_{0} \geq 3
$$

Proof. We show the case $k=1$ only. Let

$$
\begin{aligned}
\mathrm{H}_{1}^{\kappa, 1}\left(x, y_{1}\right)=2^{\kappa n} & \left(\int _ { \mathbb { R } ^ { n } } \left(\int _ { \mathbb { R } ^ { n } } \ldots \left(\int_{\mathbb{R}^{n}}\left|\mathcal{F}^{-1} \sigma_{\kappa}\left(2^{\kappa}\left(x-y_{1}\right), 2^{\kappa} x-y_{2}, \ldots, 2^{\kappa} x-y_{m}\right)\right|^{r_{m}^{\prime}}\right.\right.\right. \\
& \left.\left.\left.\times\left\langle 2^{\kappa} x-y_{m}\right\rangle^{r_{m}^{\prime} s_{m}} d y_{m}\right)^{\frac{r_{m-1}^{\prime}}{r_{m}^{\prime}}} \ldots\right)^{\frac{r_{2}^{\prime}}{r_{3}^{\prime}}}\left\langle 2^{\kappa} x-y_{2}\right\rangle^{r_{2}^{\prime} s_{2}} d y_{2}\right)^{\frac{1}{r_{2}^{\prime}}} .
\end{aligned}
$$

For any integer $j_{0} \geq 3$ and $y_{1} \in B$, we deduce from Lemma 3.1 that

$$
\begin{aligned}
& \left(\int_{S_{j_{0}}(B)}\left|\mathrm{H}_{1}^{\kappa, 1}\left(x, y_{1}\right)\right|^{r_{1}^{\prime}} d x\right)^{\frac{1}{r_{1}^{\prime}}} \\
& =2^{\kappa n}\left(\int _ { S _ { j _ { 0 } } ( B ) } \left(\int _ { \mathbb { R } ^ { n } } \left(\cdots \left(\int_{\mathbb{R}^{n}}\left|\mathcal{F}^{-1} \sigma_{\kappa}\left(2^{\kappa}\left(x-y_{1}\right), 2^{\kappa} x-y_{2}, \ldots, 2^{\kappa} x-y_{m}\right)\right|^{r_{m}^{\prime}}\right.\right.\right.\right. \\
& \left.\left.\left.\times\left\langle 2^{\kappa} x-y_{m}\right\rangle^{r_{m}^{\prime} s_{m}} d y_{m}\right)^{\frac{r_{m-1}^{\prime}}{r_{m}^{\prime}}} \ldots\right)^{\frac{r_{2}^{\prime}}{r_{3}^{\prime}}}\left\langle 2^{\kappa} x-y_{2}\right\rangle^{r_{2}^{\prime} s_{2}} d y_{2}\right)^{\frac{r_{1}^{\prime}}{r_{2}^{\prime}}} \\
& \left.\times\left|2^{\kappa}\left(x-y_{1}\right)\right|^{r_{1}^{\prime} s_{1}} d x\right)^{\frac{1}{r_{1}^{\prime}}}\left(2^{\kappa} 2^{j_{0}} R\right)^{-s_{1}} \\
& \lesssim 2^{\kappa n}\left(\int _ { \mathbb { R } ^ { n } } \left(\int_{\mathbb{R}^{n}}\left(\ldots\left(\int_{\mathbb{R}^{n}}\left|\mathcal{F}^{-1} \sigma_{\kappa}\left(z_{1}, \ldots, z_{m}\right)\right|^{r_{m}^{\prime}}\left\langle z_{m}\right\rangle^{r_{m}^{\prime} s_{m}} d z_{m}\right)^{\frac{r_{m-1}^{\prime}}{r_{m}^{\prime}}} \ldots\right)^{\frac{r_{2}^{\prime}}{r_{3}^{\prime}}}\right.\right. \\
& \left.\left.\times\left\langle z_{2}\right\rangle^{r_{2}^{\prime} s_{2}} d z_{2}\right)^{\frac{r_{1}^{\prime}}{r_{2}^{\prime}}}\left\langle z_{1}\right\rangle^{r_{1}^{\prime} s_{1}} d z_{1}\right)^{\frac{1}{r_{1}^{\prime}}} 2^{-\kappa n / r_{1}^{\prime}}\left(2^{\kappa} 2^{j_{0}} R\right)^{-s_{1}} \\
& \lesssim 2^{-\kappa\left(s_{1}-n / r_{1}\right)}\left(2^{j_{0}} R\right)^{-s_{1}} \text {. }
\end{aligned}
$$

Since $r_{l} s_{l}>n$, Hölder's inequality leads to

$$
\int_{\left(\mathbb{R}^{n}\right)^{m}}\left|\mathcal{F}^{-1} \widetilde{\sigma}_{\kappa}\left(x-y_{1}, \ldots, x-y_{m}\right)\right|\left|f_{1}\left(y_{1}\right) \ldots f_{m}\left(y_{m}\right)\right| d \vec{y}
$$




$$
\begin{aligned}
= & 2^{\kappa m n} \int_{\left(\mathbb{R}^{n}\right)^{m}}\left|\mathcal{F}^{-1} \sigma_{\kappa}\left(2^{\kappa}\left(x-y_{1}\right), \ldots, 2^{\kappa}\left(x-y_{m}\right)\right)\right|\left|f_{1}\left(y_{1}\right) \ldots f_{m}\left(y_{m}\right)\right| d \vec{y} \\
\lesssim & 2^{\kappa m n} \int_{\mathbb{R}^{n}}\left(\int _ { \mathbb { R } ^ { n } } \left(\int _ { \mathbb { R } ^ { n } } \ldots \left(\int_{\mathbb{R}^{n}}\left|\mathcal{F}^{-1} \sigma_{\kappa}\left(2^{\kappa}\left(x-y_{1}\right), \ldots, 2^{\kappa}\left(x-y_{m}\right)\right)\right|^{r_{m}^{\prime}}\right.\right.\right. \\
& \left.\left.\left.\times\left\langle 2^{\kappa}\left(x-y_{m}\right)\right\rangle^{r_{m}^{\prime} s_{m}} d y_{m}\right)^{\frac{r_{m}^{\prime}-1}{r_{m}^{\prime}}} \ldots\right)^{\frac{r_{2}^{\prime}}{r_{3}^{\prime}}}\left\langle 2^{\kappa}\left(x-y_{2}\right)\right\rangle^{r_{2}^{\prime} s_{2}} d y_{2}\right)^{\frac{1}{r_{2}^{\prime}}}\left|f_{1}\left(y_{1}\right)\right| d y_{1} \\
& \times \prod_{l=2}^{m}\left(\int_{\mathbb{R}^{n}} \frac{\left|f_{l}\left(y_{l}\right)\right|^{r_{l}}}{\left\langle 2^{\kappa}\left(x-y_{l}\right)\right\rangle^{r_{l} s_{l}}} d y_{l}\right)^{1 / r_{l}} \\
\lesssim & \int_{\mathbb{R}^{n}} \mathrm{H}_{1}^{\kappa, 1}\left(x, y_{1}\right)\left|f_{1}\left(y_{1}\right)\right| d y_{1} \prod_{l=2}^{m} M_{r_{l}} f_{l}(x) .
\end{aligned}
$$

We can also verify that for $y_{1}^{\prime} \in B$,

$$
\begin{gathered}
\int_{\left(\mathbb{R}^{n}\right)^{m}}\left|\mathcal{F}^{-1} \widetilde{\sigma}_{\kappa}\left(x-y_{1}^{\prime}, \ldots, x-y_{m}\right)\right|\left|f_{1}\left(y_{1}\right) \ldots f_{m}\left(y_{m}\right)\right| d \vec{y} \\
\lesssim \mathrm{H}_{1}^{\kappa, 1}\left(x, y_{1}^{\prime}\right) \int_{\mathbb{R}^{n}}\left|f_{1}\left(y_{1}\right)\right| d y_{1} \prod_{l=2}^{m} M_{r_{l}} f_{l}(x)
\end{gathered}
$$

and

$$
\left(\int_{S_{j_{0}}(B)}\left|\mathrm{H}_{1}^{\kappa, 1}\left(x, y_{1}^{\prime}\right)\right|^{r_{1}} d x\right)^{\frac{1}{r_{1}}} \lesssim \frac{2^{-\kappa\left(s_{k}-n / r_{k}\right)}}{\left(2^{j_{0}} R\right)^{s_{k}}} \quad \text { for integer } j_{0} \geq 3 .
$$

Taking $\mathrm{H}_{1}^{\kappa}\left(x, y_{1}, y_{1}^{\prime}\right)=\mathrm{H}_{1}^{\kappa, 1}\left(x, y_{1}\right)+\mathrm{H}_{1}^{\kappa, 1}\left(x, y_{1}^{\prime}\right)$, we complete the proof.

Lemma 3.5. Let $m$ and $k$ be positive integers with $k \leq m, \sigma$ be a multiplier satisfying (1.7) for some $s_{1}, \ldots, s_{m} \in(n / 2, n]$, and $r_{1}, \ldots, r_{m} \in(1,2]$. Then, for any ball $B$ with radial $R$, $x, x^{\prime} \in \frac{1}{4} B$, and nonnegative integers $j_{1}, \ldots, j_{m}$,

$$
\begin{aligned}
& \left(\int_{S_{j_{1}}(B)}\left(\int_{S_{j_{2}}(B)} \ldots\left(\int_{S_{j_{m}}(B)}\left|W_{0, \kappa}\left(x, y_{1}, \ldots, y_{m} ; x^{\prime}\right)\right|^{r_{m}^{\prime}} d y_{m}\right)^{\frac{r_{m-1}^{\prime}}{r_{m}^{\prime}}} \ldots\right)^{\frac{r_{1}^{\prime}}{r_{2}^{\prime}}} d y_{1}\right)^{\frac{1}{r_{1}^{\prime}}} \\
& \quad \lesssim R \frac{2^{-\kappa\left(s_{1}+\cdots+s_{m}-n / r_{1}-\cdots-n / r_{m}-1\right)}}{\prod_{1 \leq i \leq m}\left(2^{j_{i}} R\right)^{s_{i}}}
\end{aligned}
$$

provided $2^{\kappa} R<1$.

This lemma can be obtained by the argument used in the proof of Lemma 3.3 .

Lemma 3.6. Let $m$ and $k$ be positive integers with $k \leq m$, and $\sigma$ be a multiplier satisfying (1.7) for some $s_{1}, \ldots, s_{m} \in(n / 2, n]$. Suppose that $r_{1}, \ldots, r_{m} \in(1,2]$ such that $r_{k}>n / s_{k}$ for $k=1, \ldots, m$. Then, for any $B$ with radial $R, x, x^{\prime} \in B$, integer $j_{k} \geq 2$, and functions $f_{1}, \ldots, f_{m}$ satisfying supp $f_{k} \subset \mathbb{R}^{n} \backslash 4 B$ for some $k \in\{1, \ldots, m\}$,

$$
\int_{S_{j_{k}}(B)}\left(\int_{\left(\mathbb{R}^{n}\right)^{m-1}}\left|W_{0, \kappa}\left(x, y_{1}, \ldots, y_{m} ; x^{\prime}\right)\right| \prod_{l=1}^{m}\left|f_{l}\left(y_{l}\right)\right| d y_{1} \ldots d y_{k-1} d y_{k+1} d y_{m}\right) d y_{k}
$$




$$
\lesssim \frac{2^{-\kappa\left(s_{k}-n / r_{k}\right)}}{\left(2^{j_{k}} R\right)^{s_{k}-n / r_{k}}} \prod_{l=1}^{m}\left(M_{r_{l}} f_{l}(x)+M_{r_{l}} f_{l}\left(x^{\prime}\right)\right) .
$$

Proof. We consider the case $k=1$ only. As in the proof of Lemma 3.4, we have

$$
\begin{aligned}
& \int_{S_{j_{1}}(B)}\left(\int_{\left(\mathbb{R}^{n}\right)^{m-1}}\left|\mathcal{F}^{-1} \widetilde{\sigma}_{\kappa}\left(x-y_{1}, \ldots, x-y_{m}\right)\right| \prod_{l=2}^{m}\left|f_{l}\left(y_{l}\right)\right| d y_{2} \ldots d y_{m}\right)\left|f_{1}\left(y_{1}\right)\right| d y_{1} \\
& \quad \lesssim \frac{2^{-\kappa\left(s_{1}-n / r_{1}\right)}}{\left(2^{j_{1}} R\right)^{s_{1}-n / r_{1}}} \prod_{l=1}^{m} M_{r_{l}} f_{l}(x)
\end{aligned}
$$

and

$$
\begin{aligned}
& \int_{S_{j_{1}}(B)}\left(\int_{\left(\mathbb{R}^{n}\right)^{m-1}}\left|\mathcal{F}^{-1} \widetilde{\sigma}_{\kappa}\left(x^{\prime}-y_{1}, \ldots, x^{\prime}-y_{m}\right)\right| \prod_{l=2}^{m}\left|f_{l}\left(y_{l}\right)\right| d y_{2} \ldots d y_{m}\right)\left|f_{1}\left(y_{1}\right)\right| d y_{1} \\
& \quad \lesssim \frac{2^{-\kappa\left(s_{1}-n / r_{1}\right)}}{\left(2^{j_{1}} R\right)^{s_{1}-n / r_{1}}} \prod_{l=1}^{m} M_{r_{l}} f_{l}\left(x^{\prime}\right) .
\end{aligned}
$$

So we get the desired conclusion directly.

We return to show Theorem 1.2 ,

Proof of Theorem 1.2. We will employ the argument given in [16, p. 350]. For $N \in \mathbb{N}$, let

$$
\sigma^{N}\left(\xi_{1}, \ldots, \xi_{m}\right)=\sum_{|\kappa| \leq N} \widetilde{\sigma}_{\kappa}\left(\xi_{1}, \ldots, \xi_{m}\right)
$$

and denote by $T_{\sigma, N}$ the multiplier operator associated with $\sigma^{N}$. It is obvious that $T_{\sigma, N}$ is an $m$-linear singular integral operator with kernel

$$
K^{N}\left(x ; y_{1}, \ldots, y_{m}\right)=\mathcal{F}^{-1} \sigma^{N}\left(x-y_{1}, \ldots, x-y_{m}\right)
$$

in the sense of (1.1). Note that for $f_{1}, \ldots, f_{m} \in \mathscr{S}\left(\mathbb{R}^{n}\right)$,

$$
\left\|T_{\sigma}\left(f_{1}, \ldots, f_{m}\right)-T_{\sigma, N}\left(f_{1}, \ldots, f_{m}\right)\right\|_{L^{\infty}\left(\mathbb{R}^{n}\right)} \lesssim\left\|\left(\sigma-\sigma^{N}\right) \widehat{f}_{1} \ldots \widehat{f_{m}}\right\|_{L^{1}\left(\mathbb{R}^{n}\right)} \rightarrow 0 .
$$

as $N \rightarrow \infty$. By a density argument, it suffices to prove that the conclusions of Theorem 1.2 are true for $T_{\sigma, N}$ with bound independent of $N$.

Let $t_{k}=n / s_{k}$. We only need to show that, when $\sigma$ satisfies (1.7) for $s_{1}, \ldots, s_{m} \in(n / 2, n]$, all of the assumptions (i) -(iv) in Theorem 1.1 hold for the operator $T_{\sigma, N}$ provided, for $k=1, \ldots, m$, each $r_{k} \in\left(t_{k}, 2\right)$ closes enough to $t_{k}$ (satisfy $n / r_{k}>s_{k}-1 / m$ ). By Lemma 3.1, for $x \in \mathbb{R}^{n}$ and integers $j_{1}, \ldots j_{m} \in\{1,2\}$,

$$
\left(\int_{S_{j_{1}}(B(x, R))}\left(\cdots\left(\int_{S_{j_{m}}(B(x, R))}\left|\mathcal{F}^{-1} \widetilde{\sigma}_{\kappa}\left(x-y_{1}, \ldots, x-y_{m}\right)\right|^{r_{m}^{\prime}} d y_{m}\right)^{\frac{r_{m-1}^{\prime}}{r_{m}^{\prime}}} \ldots\right)^{\frac{r_{1}^{\prime}}{r_{2}^{\prime}}} d y_{1}\right)^{\frac{1}{r_{1}^{\prime}}}
$$




$$
\begin{aligned}
& \lesssim\left(\int_{S_{j_{1}}\left(B_{R}\right)}\left(\cdots\left(\int_{S_{j_{m}}\left(B_{R}\right)}\left|\mathcal{F}^{-1} \widetilde{\sigma}_{\kappa}\left(z_{1}, \ldots, z_{m}\right)\right|^{r_{m}^{\prime}}\left\langle z_{m}\right\rangle^{r_{m}^{\prime} \frac{n}{r_{m}}} d z_{m}\right)^{\frac{r_{m-1}^{\prime}}{r_{m}^{\prime}}} \ldots\right)^{\frac{r_{1}^{\prime}}{r_{2}^{\prime}}}\right. \\
& \left.\quad \times\left\langle z_{1}\right\rangle^{r_{1}^{\prime} \frac{n}{r_{1}}} d z_{1}\right)^{\frac{1}{r_{1}^{\prime}}} R^{-n / r_{1}-\cdots-n / r_{m}} \\
& \lesssim 2^{\kappa n\left(1 / r_{1}+\cdots+1 / r_{m}\right)}\left\|\sigma_{\kappa}\right\|_{W^{n / r_{1}, \ldots n / r_{m}\left(\mathbb{R}^{m n}\right)}} R^{-n / r_{1}-\cdots-n / r_{m}},
\end{aligned}
$$

which implies

$$
\begin{aligned}
& \left(\int_{A_{R}^{x}}\left(\int_{A_{R}^{x}} \ldots\left(\int_{A_{R}^{x}}\left|K^{N}\left(x ; y_{1}, \ldots, y_{m}\right)\right|^{r_{m}^{\prime}} d y_{m}\right)^{\frac{r_{m-1}^{\prime}}{r_{m}^{\prime}}} \ldots\right)^{\frac{r_{1}^{\prime}}{r_{2}^{\prime}}} d y_{1}\right)^{\frac{1}{r_{1}^{\prime}}} \\
& \quad \lesssim 2^{n N\left(1 / r_{1}+\cdots+1 / r_{m}\right)} R^{-n / r_{1}-\cdots-n / r_{m}}
\end{aligned}
$$

and hence $T_{\sigma, N}$ satisfies assumption (i) of Theorem 1.1. Denote

$$
W_{k}^{N}\left(x, y_{1}, \ldots, y_{m} ; y_{k}^{\prime}\right)=K^{N}\left(x ; y_{1}, \ldots, y_{m}\right)-K^{N}\left(x ; y_{1}, \ldots, y_{k}^{\prime}, \ldots, y_{m}\right)
$$

for $k=1, \ldots, m$, and

$$
W_{0}^{N}\left(x, y_{1}, \ldots, y_{m} ; x^{\prime}\right)=K^{N}\left(x ; y_{1}, \ldots, y_{m}\right)-K^{N}\left(x^{\prime} ; y_{1}, \ldots, y_{m}\right)
$$

Let $B$ be a ball with radial $R$. For $x, x^{\prime} \in B$ and for functions $f_{1}, \ldots, f_{m}$ with $\operatorname{supp} f_{k} \subset \mathbb{R}^{n} \backslash 4 B$, it follows from Lemmas 3.5 and 3.6 that

$$
\begin{aligned}
\int_{\left(\mathbb{R}^{n}\right)^{m}} & \left|W_{0}^{N}\left(x, y_{1}, \ldots, y_{m}, x^{\prime}\right)\right|\left|f_{1}\left(y_{1}\right) \ldots f_{m}\left(y_{m}\right)\right| d \vec{y} \\
\lesssim & \sum_{\kappa: 2^{\kappa} R>1} \sum_{j_{k}=2}^{\infty} \int_{S_{j_{k}}(B)} \int_{\left(\mathbb{R}^{n}\right)^{m-1}}\left|W_{0, \kappa}\left(x, y_{1}, \ldots, y_{m}, x^{\prime}\right)\right|\left|f_{1}\left(y_{1}\right) \ldots f_{m}\left(y_{m}\right)\right| d \vec{y} \\
& +\sum_{\kappa: 2^{\kappa} R \leq 1} \sum_{j_{1}, \ldots, j_{m}=0}^{\infty}\left(\int _ { S _ { j _ { 1 } } ( B ) } \left(\ldots\left(\int_{S_{j_{m}}(B)}\left|W_{0, \kappa}\left(x, y_{1}, \ldots, y_{m} ; x^{\prime}\right)\right|^{r_{m}^{\prime}} d y_{m}\right)^{\frac{r_{m-1}^{\prime}}{r_{m}^{\prime}}}\right.\right. \\
& \left.\ldots)^{r_{1}^{\prime}} d y_{1}\right)^{\frac{1}{r_{1}^{\prime}}} \prod_{k=1}^{m} M_{r_{l}} f_{l}(x) \prod_{l=1}^{m}\left|S_{j_{l}}(B)\right|^{\frac{1}{r_{l}}} \\
\lesssim & \sum_{\kappa: 2^{\kappa} R>1}\left(2^{\kappa} R\right)^{-s_{k}+n / r_{k}} \prod_{l=1}^{m}\left(M_{r_{l}} f_{l}(x)+M_{r_{l}} f_{l}\left(x^{\prime}\right)\right) \\
& +\sum_{\kappa: 2^{\kappa} R \leq 1}\left(2^{\kappa} R\right)^{-s_{1}-\cdots-s_{m}+n / r_{1}+\cdots+n / r_{m}+1} \prod_{l=1}^{m} M_{r_{l}} f_{l}(x) \\
\lesssim & \prod_{l=1}^{m}\left(M_{r_{l}} f_{l}(x)+M_{r_{l}} f_{l}\left(x^{\prime}\right)\right),
\end{aligned}
$$


since $s_{1}+\cdots+s_{m}<n / r_{1}+\cdots+n / r_{m}+1$. Thus, $T_{\sigma, N}$ satisfies assumption (ii) of Theorem 1.1. For a fixed integer $k \in\{1, \ldots, m\}$, a ball $B$ with radial $R$, and $x, y_{k}, y_{k}^{\prime} \in \mathbb{R}^{n}$, set

$$
\begin{aligned}
\mathrm{H}_{k, B}\left(x, y_{k}, y_{k}^{\prime}\right)= & \sum_{\kappa: 2^{\kappa} R<1}\left(\int_{\mathbb{R}^{n}} \ldots\left(\int_{\mathbb{R}^{n}}\left|W_{k, \kappa}\left(x, y_{1}, \ldots, y_{m} ; y_{k}^{\prime}\right)\right|^{r_{m}^{\prime}} d y_{m}\right)^{\frac{r_{m-1}^{\prime}}{r_{m}^{\prime}}} \ldots d y_{1}\right)^{\frac{1}{r_{1}^{\prime}}} \\
& +\sum_{\kappa: 2^{\kappa} R>1} \mathrm{H}_{k}^{\kappa}\left(x, y_{k}, y_{k}^{\prime}\right),
\end{aligned}
$$

where $\mathrm{H}_{k}^{\kappa}$ is the function satisfying (3.2) - (3.3) and the integral on the right hand side of equality is taken with respect to variables $y_{1}, \ldots, y_{k-1}, y_{k+1}, \ldots, y_{1}$. By Lemmas 3.3 and 3.4, we see that $T_{\sigma, N}$ satisfies assumption (iii) of Theorem 1.1. Finally, it follows from [9, Theorem 1.1] that $T_{\sigma, N}$ is bounded from $L^{2}\left(\mathbb{R}^{n}\right) \times L^{\infty}\left(\mathbb{R}^{n}\right) \times \cdots \times L^{\infty}\left(\mathbb{R}^{n}\right)$ to $L^{2}\left(\mathbb{R}^{n}\right)$ provided $s_{1}, \ldots, s_{m}>n / 2$, and hence assumption (iv) holds. This finishes the proof of Theorem 1.2 .

Acknowledgement. The authors would like to thank Professor N. Tomita for helpful discussion and giving preprints $[6,9$, 14] to the authors.

\section{References}

[1] B. Anh and X. T. Duong, Weighted norm inequalities for multilinear operators and applications to multilinear Fourier multipliers, arXiv:1112.0823.

[2] M. Carrozza and A. Passarelli Di Napoli, Composition of maximal operators, Publ. Mat. 40 (1996), 397-409.

[3] R. R. Coifman and Y. Meyer, On commutators of singular integrals and bilinear singular integrals, Trans. Amer. Math. Soc. 212 (1975), 315-331.

[4] R. R. Coifman and Y. Meyer, Nonlinear harmonic analysis, operator theory and PDE, Beijing Lectures in Harmonic Analysis (Beijing, 1984), 3-45, Ann. of Math. Stud. 112, Princeton Univ. Press, Princeton, NJ, 1986.

[5] A. Córdoba and C. Fefferman, A weighted norm inequality for singular integral operators, Studia Math. 57 (1976), 97-101.

[6] M. Fujita and N. Tomita, Weighted norm inequalities for multilinear Fourier multipliers, Trans. Amer. Math. Soc., to appear.

[7] L. Grafakos, Classical and Modern Fourier Analysis, Pearson Education, Inc., Upper Saddle River, NJ, 2004.

[8] L. Grafakos and N. Kalton, Multilinear Calderón-Zygmund operators on Hardy spaces, Collect. Math. 52 (2001), 169-179.

[9] L. Grafakos, A. Miyachi, and N. Tomita, On multilinear Fourier multipliers of limited smoothness, preprint.

[10] L. Grafakos and Z. Si, The Hörmander multiplier theorem for multilinear operators, J. Reine Angewandte Math., to appear.

[11] L. Grafakos and R. H. Torres, Multilinear Calderón-Zygmund theory, Adv. Math. 165 (2002), 124164.

[12] L. Grafakos and R. H. Torres, On multilinear singular integrals of Calderón-Zygmund type, Publ. Mat. 2002, Vol. Extra, 57-91.

[13] G. Hu, Weighted norm inequalities for multilinear Calderón-Zygmund operators, Sci. China Math. 53 (2010), 1863-1876. 
[14] A. Miyachi and N. Tomita, Minimal smoothness conditions for bilinear Fourier multiplier, Rev. Mat. Iberoamericana, to appear.

[15] C. Kenig and E. M. Stein, Multilinear estimates and fractional integral, Math. Res. Lett. 6 (1999), $1-15$.

[16] D. S. Kurtz and R. L. Wheeden, Results on weighted norm inequalities for multipliers, Trans. Amer. Math. Soc. 255 (1979), 343-362.

[17] A. Lerner, S. Ombrossi, C. Perez, R. H. Torres, and R. Trojillo-Gonzalez, New maximal functions and multiple weights for the multilinear Calderón-Zygmund theory, Adv. Math. 220 (2009), 12221264.

[18] N. Tomita, A Hörmander type multiplier theorem for multilinear operator, J. Funct. Anal. 259 (2010), 2028-2044. 